\title{
5 Automated Drivability und straßenräumliche Verträglichkeit im Stadt-Land-Kontinuum am Beispiel der Stadtregion Wien
}

Aggelos Soteropoulos

1. Einleitung

2. Methodik der vorliegenden Untersuchung

2.1 Ermittlung der Eignung von Straßenräumen für den Einsatz automatisierter Fahrzeuge: Automated Drivability

2.2 Vorgangsweise zur Bewertung der straßenräumlichen Verträglichkeit bei den Straßenabschnitten

3. Untersuchungsgemeinden in der Stadtregion Wiens und analysierte Straßenabschnitte

4. Ergebnisse der Untersuchung

4.1 Ergebnisse zur Eignung von Straßenräumen für den Einsatz automatisierter Fahrzeuge (Automated Drivability)

4.2 Ergebnisse zur straßenräumlichen Verträglichkeit

4.3 Zusammenschau der Ergebnisse zur Automated Drivability und straßenräumlichen Verträglichkeit

5. Diskussion und Fazit

Literatur

Aggelos Soteropoulos

TU Wien, future.lab Research Center und Forschungsbereich Verkehrssystemplanung (IVS)

aggelos.soteropoulos@tuwien.ac.at

(C) Der/die Autor(en) 2021

M. Mitteregger et al. (Hrsg.), AVENUE21. Politische und

planerische Aspekte der automatisierten Mobilität,

https://doi.org/10.1007/978-3-662-63354-0_5 


\section{EINLEITUNG}

Automatisierte Fahrzeuge werden unsere Mobilität und die Möglichkeit des Vorankommens stark verändern. Bereits in den nächsten Jahren sind hierdurch im Mobilitätsbereich hochdynamische Entwicklungen mit grundlegenden Veränderungen und Umbrüchen zu erwarten, die sowohl Chancen als auch Risiken mit sich bringen werden. Aus Sicht der Verkehrs-, Infrastruktur- und Stadtplanung braucht es daher Strategien, wo und wie automatisierte Fahrzeuge bestmöglich eingesetzt werden.

Bisher wurden bereits zahlreiche Studien zu den verkehrlichen und räumlichen Wirkungen automatisierter Fahrzeuge durchgeführt, die als Grundlage für die Entwicklung von Strategien und Maßnahmen für die politischen Entscheidungsträger dienen können. Chancen durch automatisierte Fahrzeuge ergeben sich demnach unter anderem durch eine höhere Kapazitätsausnutzung der vorhandenen Verkehrsinfrastruktur, eine verbesserte Wirtschaftlichkeit des öffentlichen Verkehrs (ÖV), falls die Personalkosten verringert werden können, oder durch erweiterte Mobilitätsoptionen für spezifische Nutzergruppen, wie z. B. Mobilitätseingeschränkte (vgl. Milakis et al. 2017: 13; Soteropoulos et al. 2019a: 12). Gleichfalls gehen die Studien jedoch größtenteils ebenso davon aus, dass sich mit dem Einsatz automatisierter Fahrzeuge durch die zahlreichen Vorteile im Hinblick auf Komfort sowie durch die Erschließung neuer Nutzergruppen die Attraktivität der Fahrzeugnutzung und damit die Verkehrsleistung in den für automatisierte Fahrzeuge intendierten Bereichen erhöhen wird (vgl. Soteropoulos et al. 2019b: 40; Hörl et al. 2019: 26; Marsden et al. 2018: 31). Des Weiteren führt der durch automatisierte Fahrzeuge ermöglichte Verkehrsfluss mit kürzeren Fahrzeugfolgen - die eine höhere Kapazitätsausnutzung der Verkehrsinfrastruktur ermöglichen - zu dichterem Verkehr, wodurch Fußgängerlnnen, Radfahrerlnnen und abbiegende Fahrzeuge schwerer Lücken finden, sich also die Trennwirkung der Straßen erhöht bzw. die Durchlässigkeit des Straßenraums für andere Verkehrsteilnehmerlnnen verringert (vgl. Abegg et al. 2018: 26; Wissenschaftlicher Beirat beim Bundesminister für Verkehr und digitale Infrastruktur 2017: 23; Heinrichs 2015: 237).

Bislang behandeln die Studien jedoch nur die mögliche grobe Stoßrichtung potentieller Wirkungen. Nur unzureichend Berücksichtigung findet in diesem Zusammenhang die Ebene des Straßenraums. Der Straßenraum ist öffentlicher Raum, d. h. grundsätzlich für alle jederzeit zugänglich und im Wesentlichen im öffentlichen Eigentum, bildet aber nur eine Teilmenge des öffentlichen Raums ab, zu welchem neben dem Straßenraum auch beispielsweise Plätze, Parks, siedlungsbezogene, öffentlich zugängliche Freiräume oder Erdgeschosszonen gehören, die jeweils aber wiederum stark mit dem Straßenraum zusammenhängen (vgl. Stadt Wien 2018: 13). Die Ebene des Straßenraums ist aus zweierlei Hinsicht besonders relevant (Abb. 1):

1. Städte, aber auch ländliche Siedlungsstrukturen weisen sehr unterschiedliche Straßenräume auf, die sich hinsichtlich ihrer Funktion (z. B. der Verbindungs- oder Erschließungsfunktion), der Verkehrsmengen, Gebietscharakteristik (z. B. alter Dorfkern oder neues Gewerbegebiet), Umfeldnutzungen sowie straßenräumlicher Situationen (z. B. Begrenzung, Breite und Verlauf) unterscheiden (vgl. Marshall 2005: 54; Baier/FGSV 2007: 5). Für den Einsatz automatisierter Fahrzeuge bedeuten diese unterschiedlichen Straßenraumkontexte unterschiedliche Anforderungen: Nimmt die Komplexität der Einsatzumgebung (Operational Design Domain - ODD) beispielsweise durch eine Vielzahl von zu erfassenden Fußgängerlnnen, RadfahrerInnen oder durch komplexe Knotenpunktformen zu, so steigen die Ansprüche an das automatisierte Fahrsystem (vgl. Czarnecki 2018; SAE International 2018: 14). Für die Verkehrs-, Infrastruktur- und Stadtplanung kann die Analyse von Straßenräumen hinsichtlich der Eignung für automatisierte Fahrzeuge 
aus technisch-infrastruktureller Sicht daher Informationen darüber geben, wo automatisierte Fahrzeuge aufgrund der umgebenden Straßenraum- und Umfeldbedingungen vermutlich eher bzw. mit weniger Infrastrukturadaptierungen verkehren können und wo nicht. Damit kann auch erkannt werden, bei welchen Straßenräumen der Einsatz automatisierter Fahrzeuge mit größerem oder weniger großem Aufwand möglich ist.

2. An Straßenräume besteht grundsätzlich eine Fülle von Nutzungsansprüchen, Anforderungen und Bedürfnissen von Menschen, sich darin aufzuhalten oder zu bewegen. Neben der Verkehrsfunktion von Straßen ergeben sich die Art und das Ausmaß weiterer Anforderungen an den Straßenraum aus dem Straßenumfeld, d. h. aus der Art der Nutzung der Randbebauung, deren Orientierung zum Straßenraum und anderen Faktoren (vgl. Bühlmann/ Laube 2013: 9). Diese stehen zum Teil in Widerspruch zueinander und führen zu Nutzungskonflikten (vgl. Häfliger et al. 2015: 19). Insbesondere Konflikte zwischen den Ansprüchen des motorisierten Individualverkehrs und den übrigen Bedürfnissen (andere Verkehrsarten wie Zufußgehen und Radfahren) sind nur bis zu einer gewissen Intensität verträglich bzw. abhängig von der konkreten Situation zumutbar. Steigt die Verkehrsbelastung jedoch über diese Grenze, erreicht sie eine Dominanz, welche die übrigen Nutzungsansprüche in nicht mehr verträglichem Ausmaß beeinträchtigt (vgl. Bühlmann/Laube 2013: 10). Aus Sicht der Planung ist es deshalb ebenso notwendig, zu untersuchen, inwieweit der mögliche Einsatz automatisierter Fahrzeuge aufgrund der mit innen verbundenen Wirkungen wie einer Zunahme der Verkehrsleistung sowie einer Erhöhung der Trennwirkung durch eine dichtere Fahrzeugfolge mit den übrigen Nutzungsansprüchen an den Straßenraum verträglich ist.

Abbildung 1: Überblick zur Relevanz der Straßenräume für den Einsatz automatisierter Fahrzeuge

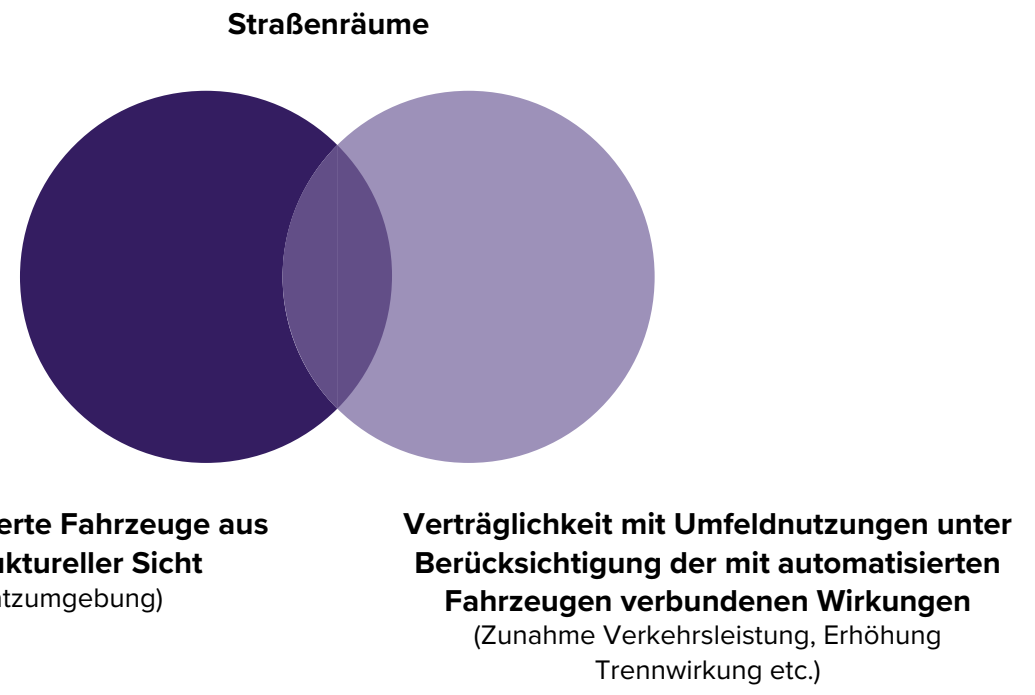

Quelle: eigene Darstellung

Im Rahmen des Beitrags soll daher einerseits die Eignung von Straßenräumen für automatisierte Fahrzeuge im Stadt-Land-Kontinuum am Beispiel von vier verschiedenen Gemeinden in der Stadtregion Wien untersucht werden. Hierbei werden verschiedene Ausprägungen von Kriterien (z. B. Kreuzungen, Geschwindigkeitslimits etc.), die sich aus den aktuellen Problemstellungen automatisierter Fahrsysteme aus Testberichten (vgl. BMVIT 2018, Favarò et al. 2018) ergeben, miteinander verknüpft, um die Eignung der Straßenabschnitte für den funktionierenden Einsatz automatisierter Fahrsysteme abzubilden. Andererseits werden im Rahmen einer Analyse der straßenräumlichen Verträglichkeit beispielhaft gleichfalls die Konflikte, die sich aus dem derzeitigen Kfz-Verkehr (Verkehrsmenge, Geschwindigkeit) für Fußgängerlnnen und Radfahrerlnnen im 
Längsverkehr, beim Aufenthalt und bei Fahrbahnüberquerungen ergeben, bewertet (vgl. Baier et al. 2011: 37). Hier wird an einzelnen Straßenabschnitten in den vier Untersuchungsgemeinden untersucht, ob und wie sich in Straßenräumen schon heute Probleme wie eine hohe Trennwirkung bzw. eine geringe Durchlässigkeit für Fußgängerlnnen und Radfahrerlnnen zeigen, indem explizit Kriterien wie die Verkehrsstärke, die Geschwindigkeit der Fahrzeuge oder der Anteil an Lkws in der Analyse untersucht und berücksichtigt werden (vgl. Nørby/Meltofte 2012: Vl; Litman 2009: 1). Anschließend wird die Bewertung der straßenräumlichen Verträglichkeit mit der Bewertung der Eignung des Straßenraums für automatisierte Fahrsysteme verknüpft bzw. gemeinsam betrachtet. Es geht also darum, exemplarisch zu untersuchen, inwieweit die Eignung von StraBenräumen für automatisierte Fahrzeuge und die straßenräumliche Verträglichkeit ebendieser Straßenräume zusammenwirken (Abb. 2). Die Zusammenführung beider Bewertungen ist von wesentlicher Bedeutung, um abzuschätzen, wo aus verkehrs- und stadtplanerischer Sicht in naher Zukunft besonderer Handlungsbedarf besteht, sowie um entsprechende Strategien für einen bestmöglichen Einsatz automatisierter Fahrzeuge zu entwickeln.

Abbildung 2: Zusammenhang zwischen der Eignung von Straßenräumen für automatisierte Fahrzeuge aus technisch-infrastruktureller Sicht und straßenräumlicher Verträglichkeit

Straßenräumliche Verträglichkeit (mit Umfeldnutzungen)

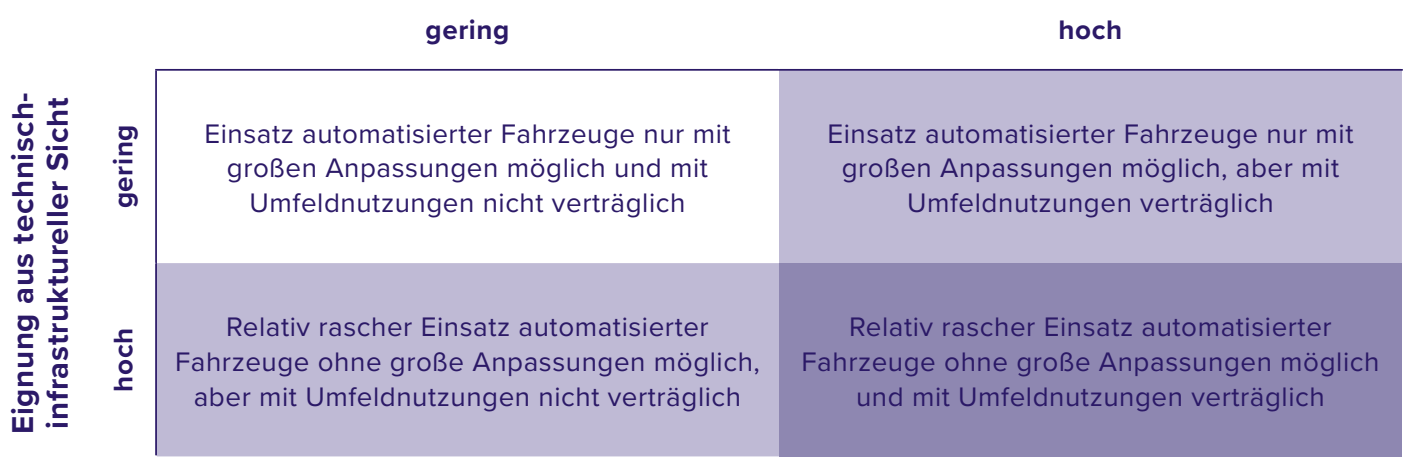

Quelle: eigene Darstellung

\section{METHODIK DER VORLIEGENDEN UNTERSUCHUNG}

Zur Ermittlung der Eignung von Straßenräumen für den Einsatz automatisierter Fahrzeuge aus technisch-infrastruktureller Sicht sowie zur Bewertung der straßenräumlichen Verträglichkeit wurden umfangreiche Verfahren angewendet, die im Folgenden erläutert werden.

\subsection{ERMITTLUNG DER EIGNUNG VON STRASSENRÄUMEN FÜR DEN EINSATZ AUTOMATISIERTER FAHRZEUGE: AUTOMATED DRIVABILITY}

Die Methodik für die Ausarbeitung der Bewertung der Eignung von Straßenräumen für den Einsatz automatisierter Fahrzeuge beruht sehr stark auf dem in der Arbeit von Soteropoulos et al. (2020) beschriebenen Konzept der Automated Drivability. Im Folgenden wird die angewandte Methodik nur kurz darlegt, für eine ausführliche Beschreibung wird auf Soteropoulos et al. (2020) verwiesen. 


\subsubsection{Ausgangspunkt, Framework und Komponenten der Automated Drivability}

Abbildung 3: Zentrale Aufgaben eines automatisierten Fahrsystems und Komponenten des Framework für die Bewertung der Automated Drivability inklusive Subelemente

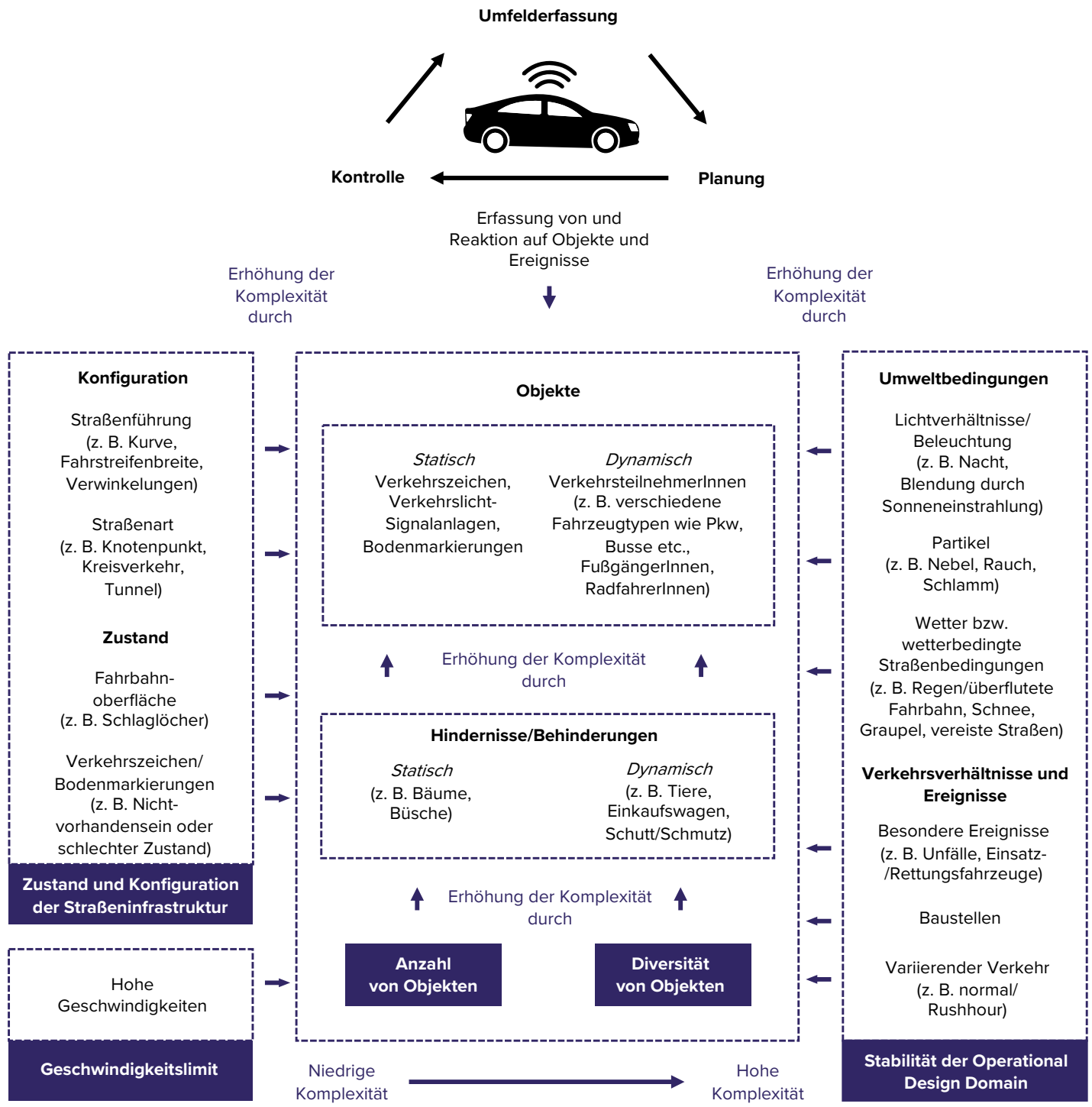

Quelle: eigene Darstellung

Das Konzept der Automated Drivability geht davon aus, dass bestimmte straßenräumliche Kontexte sowie Umfeldbedingungen (ODD) die Anforderungen an automatisierte Fahrsysteme zur Ausführung ihrer Fahraufgabe erhöhen. Prozesse wie (1) die Umfelderfassung und -abbildung, (2) die Planung und das Treffen einer entsprechenden Fahrentscheidung sowie (3) die Ausführung der entsprechenden Fahrentscheidung (Kontrolle), welche für das Ausführen der Fahraufgabe durch das automatisierte Fahrzeug notwendig sind, können durch verschiedene Faktoren und Rahmenbedingungen erschwert werden. Darauf aufbauend wurde ein Framework zur Beschreibung der Automated Drivability mit verschiedenen Komponenten und 
Subelementen, die die Komplexität für automatisierte Fahrsysteme erhöhen, entwickelt. Das Framework umfasst die folgenden Komponenten:

1. Anzahl der Objekte im Straßenraum

2. Diversität der Objekte im Straßenraum

3. Zustand und Konfiguration der Straßeninfrastruktur

4. Geschwindigkeitslimit

5. Stabilität der Operational Design Domain

Abbildung 3 (auf der vorigen Seite) gibt einen Überblick zu zentralen Aufgaben eines automatisierten Fahrsystems sowie die Komponenten des Framework zur Bewertung der Automated Drivability inklusive der Subelemente.

\subsubsection{Ableitung von Indikatoren für die verschiedenen Komponenten des Framework}

Für jede der eingangs beschriebenen Komponenten des Framework der Automated Drivability werden in weiterer Folge unter Berücksichtigung (öffentlich) verfügbarer Daten geeignete Indikatoren abgeleitet. Für die Berechnung der Indikatoren wurden im Rahmen dieser Untersuchung Daten der österreichischen Graphenintegrationsplattform (GIP), der Open Street Map (OSM) sowie der Österreichischen Anstalt für Meteorologie und Geodynamik (ZAMG) herangezogen. Die Berechnung wurde mit Hilfe der Geoinformationssystemsoftware ArcGIS durchgeführt. Tabelle 1 (siehe nächste Seite) gibt einen Überblick der abgeleiteten Indikatoren für die jeweiligen Komponenten sowie die jeweiligen Datenquellen.

\subsubsection{Verknüpfung der Indikatoren}

Zur Verknüpfung der Indikatoren werden die Werte für jeden Indikator zunächst mit Hilfe folgender Formel normiert und standardisiert:

$$
X_{i j}^{\prime}= \begin{cases}\frac{X_{i j}-\min X_{i j}}{\max X_{i j}-\min X_{i j}}, & \text { positiv } \\ \frac{\max X_{i j}-X_{i j}}{\max X_{i j}-\min X_{i j}}, & \text { negativ }\end{cases}
$$

Dabei sind

- $\quad \mathrm{X}_{\mathrm{ij}}$ der Wert des Indikators i für den Straßenabschnitt j und

- $\quad \max _{i j}$ und $\min X_{i j}$ die maximalen bzw. minimalen Werte des Indikators i für alle Straßenabschnitte im Untersuchungsgebiet.

Bei positiven Indikatoren handelt es sich um jene Indikatoren, bei denen ein höherer Wert des Indikators eine höhere Automated Drivability darstellt (z. B. Breite der Straße), während es sich bei negativen Indikatoren um jene handelt, bei denen ein geringerer Wert des Indikators eine höhere Automated Drivability bedeutet (z. B. Anzahl von Ampeln; siehe auch Tab. 1). 


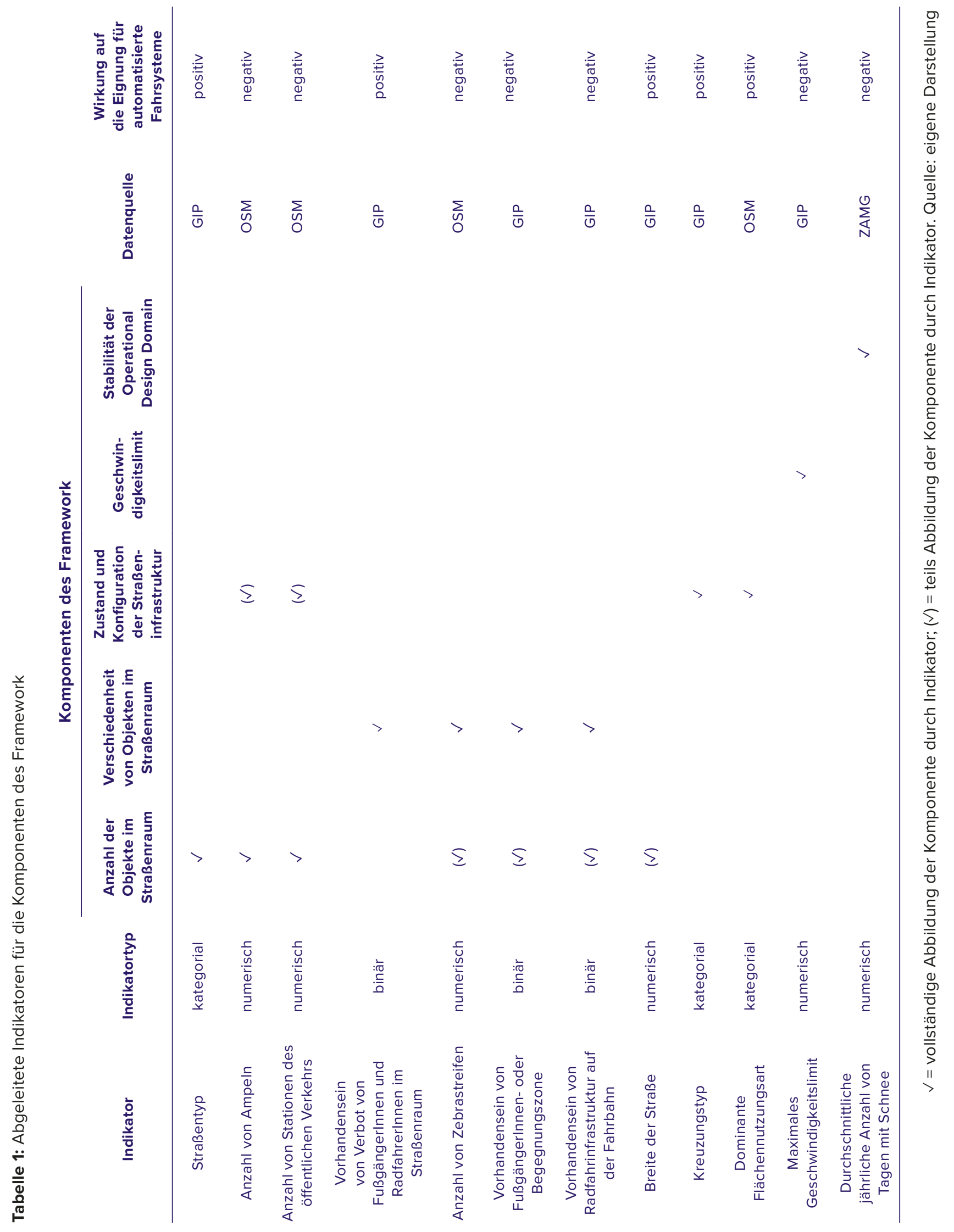


Anschließend wurde eine Verknüpfung der Indikatoren mittels Gewichtung einzelner Komponenten vorgenommen. Ausgehend von Einschätzungen über die Relevanz der einzelnen Komponenten hinsichtlich der Komplexität für automatisierte Fahrsysteme in der Literatur (vgl. Pendleton 2017; Brummelen et al. 2018; Shladover 2018a, 2018b; Favarò et al. 2018) wurde folgende Gewichtung der Komponenten vorgenommen:

1. Anzahl Objekte im Straßenraum:

2. Verschiedenheit der Objekte im Straßenraum:

3. Zustand und Konfiguration der Straßeninfrastruktur:

4. Geschwindigkeitslimit:

5. Stabilität der Operational Design Domain:

Zur Verknüpfung wurden die Indikatorwerte anschließend für jeden Straßenabschnitt unter Verwendung folgender Formel durch eine Addition der Werte des Indikators für jede Komponente des Framework summiert und durch die Anzahl der Indikatoren pro Komponente geteilt sowie mit dem jeweiligen Gewicht multipliziert, um den aggregierten Wert des Indexes für die Automated Drivability (ADX) für jeden Straßenabschnitt zu erhalten:

$$
\begin{gathered}
0.188 *\left(\left(X c 1_{i 1 j}+X c 1_{i_{2} j} \ldots\right) / I c 1_{n}\right)+0.438 *\left(\left(X c 2_{i_{1} j}+X c 2_{i_{2} j} \ldots\right) / I c 2_{n}\right)+ \\
X_{A D X j}=0.125 *\left(\left(X c 3_{i_{1} j}+X c 3_{i_{2} j} \ldots\right) / I c 3_{n}\right)+0.188 *\left(\left(X c 4_{i_{1} j}+X c 4_{i_{2} j} \ldots\right) / I c 4_{n}\right)+ \\
0.063 *\left(\left(X c 5_{i_{1} j}+X c 5_{i_{2} j} \ldots\right) / I c 5_{n}\right)
\end{gathered}
$$

Wobei

- $\quad \mathrm{X}_{\mathrm{ADXj}}$ der Wert des ADX für den Straßenabschnitt $\mathrm{j}$,

- Xc1.1j der Wert des Indikators i1 der Komponente 1,

- $\quad \mathrm{Xc2}{ }_{\mathrm{ijj}}$ der Wert des Indikators i1 der Komponente 2 ist ...; und

- $\quad$ Ic1 ${ }_{n}$ die Gesamtzahl der Indikatoren für die Komponente 1 und

- Ic2 ${ }_{n}$ die Gesamtzahl der Indikatoren für die Komponente 2 ...

darstellt.

\subsection{VORGANGSWEISE ZUR BEWERTUNG DER STRASSENRÄUMLICHEN VERTRÄG- LICHKEIT BEI DEN STRASSENABSCHNITTEN}

Für die Bewertung der straßenräumlichen Verträglichkeit finden sich in der Literatur zahlreiche Verfahren wie beispielsweise das M.A.R.S.-Modell der autonomen und relativen Standards (vgl. Baier 1992), das LADIR-Verfahren (Müller et al. 1994) oder der Kompensatorische Ansatz (Mörner et al. 1984). Diese Verfahren haben ihren Ursprung in den 1980er und 1990er Jahren, sie kamen seither jedoch zum Teil in adaptierter Form auch in den letzten Jahren zum Beispiel bei Bühlmann und Laube (2013), Frehn et al. (2013) oder Baier et al. (2011) zur Anwendung. 
Als Grundlage der Bewertung der straßenräumlichen Verträglichkeit im Rahmen dieses Beitrags wurde der Kompensatorische Ansatz von Mörner et al. (1984) verwendet und mittels der Erkenntnisse der erst kürzlich erfolgten Anwendung des Ansatzes durch Bühlmann und Laube (2013) adaptiert. Beim Kompensatorischen Ansatz wird für einzelne Straßenabschnitte ausgehend von der Randbebauung bzw. der angrenzenden Nutzung sowie Funktion und Bedeutung der Ortschaft - eine maximale Verkehrsbelastung in der Dimension „Anzahl der Kraftfahrzeuge pro Spitzenstunde" (Kfz/Sp-h) festgelegt. In Abhängigkeit verschiedener Einflussfaktoren wird diese maximale Verkehrsbelastung korrigiert und auf dieser Basis dann die Beurteilung der Verträglichkeit des vorhandenen Verkehrsaufkommens mit dem Straßenumfeld (gemessene Verkehrsbelastung im Vergleich zu maximal verträglicher Verkehrsbelastung) vorgenommen. Unter Berücksichtigung der mit dem automatisierten Fahren verbundenen und vorher beschriebenen Wirkungen hinsichtlich einer Zunahme der Trennwirkung durch eine mögliche dichtere Fahrzeugfolge wurde im Rahmen des angewendeten Kompensatorischen Ansatzes ebenso der Aspekt der Trennwirkung explizit miteinbezogen bzw. der Ansatz um diesen Aspekt erweitert.

\subsubsection{Bestimmung des Grundwerts der noch verträglichen Verkehrsbelastung}

Die Bestimmung des Grundwerts der Verkehrsbelastung, d. h. die noch verträgliche Verkehrsbelastung, demnach Fahrzeuge pro Spitzenstunde (Fz/Sp-h), ergibt sich aus der angrenzenden Nutzung bzw. Randbebauung des Straßenabschnitts. Während Mörner et al. (1984) im Rahmen ihres Ansatzes eher von städtischen Gebieten ausgegangen sind, verweisen Bühlmann und Laube (2013) im Rahmen ihrer Forschungsarbeit auch auf den Zusammenhang zwischen noch verträglicher Verkehrsbelastung und der Funktion und Bedeutung der Ortschaft. Hierbei ist die Akzeptanz von Verkehr in ländlichen Gebieten geringer als in städtischen Zentren (vgl. BühImann/Laube 2013: 34). Da sich die untersuchten Straßenabschnitte allesamt in kleineren Städten im Stadtumland von Wien befinden (siehe Abschnitt 3), wurden ausgehend von Mörner et al. (1984) sowie Bühlmann und Laube (2013) die folgenden vier verschiedenen Kategorien unterschieden:

Kategorie A Einkauf/Zentrum, tendenziell mit geschlossener Blockbebauung mit mehr als zwei Geschossen und mittlerer bis intensiver Geschäftsnutzung

Kategorie B Mischnutzung, tendenziell mit offener bis halboffener zwei- bis viergeschossiger Bebauung bzw. geschlossener ein- bis zweigeschossiger Bebauung und mittlerer Geschäftsnutzung

Kategorie C Wohnen, tendenziell mit offener Ein- und Zweifamilienhausbebauung und nur vereinzelten Geschäften oder sonstigen publikumsintensiven Nutzungen

Kategorie D Gewerbe- und Industriegebiet, tendenziell mit geringen Anliegeransprüchen und keinen publikumsintensiven Nutzungen wie Wohnen oder (fußläufiges) Einkaufen

Der Grundwert der mit Anliegeransprüchen an das Straßenumfeld noch verträglichen Verkehrsbelastung ( $\mathrm{Fz} / \mathrm{Sp}-\mathrm{h})$ wurde für die vier Kategorien dabei in adaptierter Form zu Mörner et al. (1984) sowie Bühlmann und Laube (2013) wie folgt festgesetzt und geht gemäß Bühlmann und Laube (2013) von der Annahme aus, dass in kleineren Städten bzw. in ländlichen Gebieten die Akzeptanz gegenüber dem Verkehr geringer ist als in größeren Städten (vgl. ebd.: 17). Zudem wurde hierbei davon ausgegangen, dass in Zentrumsbereichen weitaus höhere Anforderungen an die Aufenthaltsqualität und somit die Verträglichkeit gestellt werden als in Gewerbe- und Industriegebieten: 
Kategorie A 150 Fahrzeuge pro Spitzenstunde

Kategorie B 250 Fahrzeuge pro Spitzenstunde

Kategorie C 400 Fahrzeuge pro Spitzenstunde

Kategorie D 1.000 Fahrzeuge pro Spitzenstunde

\subsubsection{Bestimmung der tatsächlichen noch verträglichen Verkehrsbelastung: Adap- tierung des Grundwerts durch Nutzung und Gestaltung des Straßenraums}

Die im ersten Schritt unterschiedenen Kategorien bilden nur eine grobe Aufteilung. Innerhalb der jeweiligen Kategorien kann sich jedoch die Nutzung und Gestaltung des Straßenraums deutlich unterscheiden. Daher wird der im ersten Schritt festgesetzte Grundwert der noch verträglichen Verkehrsbelastung in weiterer Folge in Abhängigkeit von verschiedenen Bewertungskriterien adaptiert. Bei diesen Bewertungskriterien handelt es sich um
a. Nutzung durch Fußgängerlnnen und Radfahrerlnnen,
b. Flächenaufteilung,
c. Grün und Gestaltung,
d. Geschwindigkeit,
e. Schwerverkehr/Lkw-Anteil und
f. Trennwirkung: Querungshilfen/Umwege für Fußverkehr.

Die Kriterien dienen als Kompensationsaspekte, mit denen sich die Höhe der verträglichen Verkehrsbelastung mit den übrigen Nutzungsansprüchen an den Straßenraum erhöht bzw. verringert. In Anlehnung an Mörner et al. (1984) und mittels geringfügiger Adaptierung dieser Werte führen die Verträglichkeitsstufen der jeweiligen Bewertungskriterien dabei zu der in Tabelle 2 ersichtlichen Kompensation bzw. Adaptierung des Grundwerts der noch verträglichen Verkehrsbelastung.

Tabelle 2: Überblick der Verträglichkeitsstufen je Bewertungskriterium und den entsprechenden Adaptierungswert für die Grundwerte der noch verträglichen Verkehrsbelastung

\begin{tabular}{cc}
\hline $\begin{array}{c}\text { Verträglichkeitsstufe des jeweiligen } \\
\text { Bewertungskriteriums }\end{array}$ & $\begin{array}{c}\text { Adaptierung des Grundwerts der noch } \\
\text { verträglichen Verkehrsbelastung um }\end{array}$ \\
\hline++ (gut verträglich) & $+50 \mathrm{Fz} / \mathrm{Sp}-\mathrm{h}$ \\
+ (verträglich) & $+25 \mathrm{Fz} / \mathrm{Sp}-\mathrm{h}$ \\
$\circ$ (gerade noch verträglich) & $\pm 0 \mathrm{Fz} / \mathrm{Sp}-\mathrm{h}$ \\
- (unverträglich) & $-25 \mathrm{Fz} / \mathrm{Sp}-\mathrm{h}$ \\
-- (völlig unverträglich) & $-50 \mathrm{Fz} / \mathrm{Sp}-\mathrm{h}$ \\
\hline
\end{tabular}


a) Nutzung durch Fußgängerlnnen und Radfahrerlnnen

Mit dem Bewertungskriterium „Nutzung durch FußgängerInnen und RadfahrerInnen“ wird von der Annahme ausgegangen, dass der Kfz-Verkehr FußgängerInnen und RadfahrerInnen in ihren Aktivitäten im Straßenraum stört bzw. gefährdet. Die Bewertung folgt daher der Logik, dass mit einer zunehmenden Anzahl von FußgängerInnen und Radfahrerlnnen, die sich auf einer Straße aufhalten, Kfz-Verkehr weniger verträglich ist. Hierzu wird die Anzahl der Fußgängerlnnen und Radfahrerlnnen (also die durch Kfz-Verkehr Belästigten bzw. Gefährdeten) in der Spitzenstunde herangezogen und infolge die in Tabelle 3 ersichtlichen Verträglichkeitsstufen festgesetzt.

Tabelle 3: Verträglichkeitsstufen für das Kriterium „Nutzung durch FußgängerInnen und RadfahrerInnen“

\begin{tabular}{cc}
\hline $\begin{array}{c}\text { Anzahl von Fußgängerlnnen und Radfah- } \\
\text { rerlnnen auf der Straße in der Spitzenstunde }\end{array}$ & $\begin{array}{c}\text { Verträglichkeitsstufen (Verträglichkeit } \\
\text { des Kfz-Verkehrs mit dieser Anzahl von } \\
\text { FußgängerInnen und RadfahrerInnen) }\end{array}$ \\
\hline$<100$ & ++ (gut verträglich) \\
über 100 bis 200 & + (verträglich) \\
über 200 bis 400 & o (gerade noch verträglich) \\
über 400 bis 600 & - (unverträglich) \\
über 600 & -- (völlig unverträglich)
\end{tabular}

Quelle: eigene Darstellung nach Mörner et al. (1984)

\section{b) Flächenaufteilung}

Mit dem Bewertungskriterium „Flächenaufteilung“ wird die Breite von Flächen für Fußgängerlnnen und Radfahrerlnnen (Fußweg-, Radweg- und Grünstreifenflächen) im Verhältnis zur Kfz-Verkehrsfläche (der Fahrverkehrsfläche inklusive Mehrzweckspuren sowie Flächen für den ruhenden Kfz-Verkehr) evaluiert. Dabei wird davon ausgegangen, dass je geringer das Verhältnis zwischen der Breite der Fläche für Fußgängerlnnen und Radfahrerlnnen im Straßenraum im Vergleich zur Breite der Kfz-Verkehrsfläche ist, desto weniger verträglich stellt sich der Kfz-Verkehr dar . Hierzu werden hinsichtlich des Verhältnisses zwischen der Breite der Fläche für FußgängerInnen und Radfahrerlnnen zur Breite der Kfz-Verkehrsflächen die in Tabelle 4 enthaltenen Verträglichkeitsstufen festgesetzt.

Tabelle 4: Verträglichkeitsstufen für das Kriterium „Flächenaufteilung“

\begin{tabular}{cc}
\hline $\begin{array}{c}\text { Verhältnis der Breite von Flächen für } \\
\text { Fußgängerlnnen und RadfahrerInnen zur } \\
\text { Breite der Kfz-Verkehrsflächen }\end{array}$ & $\begin{array}{c}\text { Verträglichkeitsstufen (mit den Anliegeran- } \\
\text { sprüchen und dem Umfeld) }\end{array}$ \\
\hline$>1,00$ & ++ (gut verträglich) \\
0,75 bis unter 1,00 & + (verträglich) \\
0,5 bis unter 0,75 & o (gerade noch verträglich) \\
0,25 bis unter 0,5 & - (unverträglich) \\
unter 0,25 & -- (völlig unverträglich) \\
\hline
\end{tabular}

Quelle: eigene Darstellung nach Mörner et al. (1984)

\section{c) Grün und Gestaltung}

Mit dem Bewertungskriterium „Grün und Gestaltung“ wird im Hinblick auf die Gestaltung eines Straßenraums mit Grünflächen und Bäumen bzw. damit zusammenhängenden gestalterischen Elemente eine Wertung vorgenommen. Hierbei wird von der Annahme ausgegangen, dass weniger Grünflächen und Bäume bzw. damit zusammenhängende gestalterische Elemente im Straßenraum die Aufenthaltsqualität verringern und damit Kfz-Verkehr weniger verträglich ist. 
Die Bewertung erfolgt hierbei qualitativ (als Ersatz für quantifizierbare Merkmale wie die Anzahl der Bäume bzw. Anzahl der Grün- und Gestaltungselemente etc.), wobei dabei die in Tabelle 5 beschriebenen Kategorien und Verträglichkeitsstufen unterschieden werden.

Tabelle 5: Verträglichkeitsstufen für das Kriterium „Grün und Gestaltung“

Grün und Gestaltung des Straßenraums
Verträglichkeitsstufen (mit den Anliegeransprüchen und dem Umfeld)

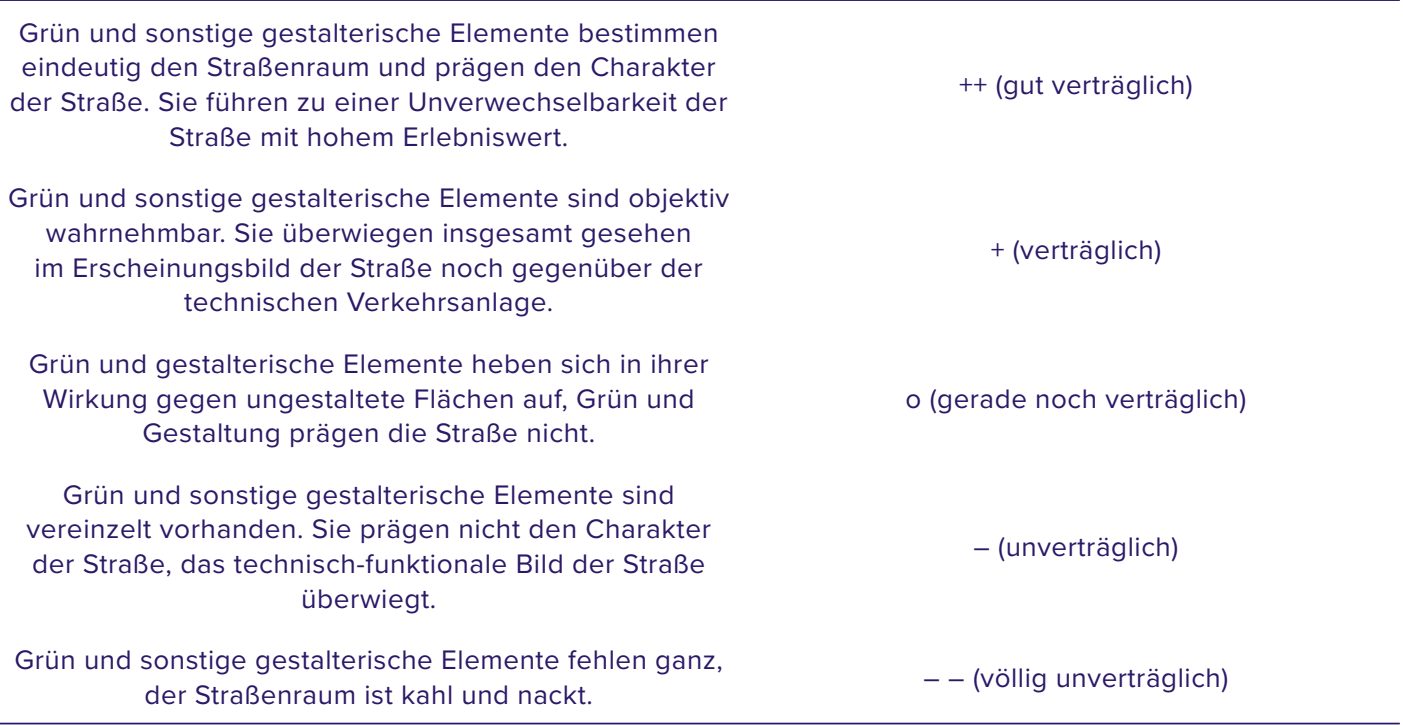

\section{d) Geschwindigkeit}

Mit dem Bewertungskriterium "Geschwindigkeit“ werden gefahrene Geschwindigkeiten auf dem Straßenabschnitt begutachtet. Es wird davon ausgegangen, dass die gefahrenen Geschwindigkeiten die Nutzbarkeit der Straßenoberfläche durch AnwohnerInnen und die Sicherheit von nichtmotorisierten StraßennutzerInnen (z. B. bezüglich der Querbarkeit der Fahrbahn) maßgeblich beeinflussen und höhere gefahrene Geschwindigkeiten auf dem Straßenabschnitt Kfz-Verkehr weniger verträglich macht. Für die Bewertung werden hierbei die gefahrenen Geschwindigkeiten in Form von v85 ${ }^{1}$ herangezogen und infolge die in Tabelle 6 ersichtlichen Verträglichkeitsstufen festgesetzt.

Tabelle 6: Verträglichkeitsstufen für das Kriterium „Geschwindigkeit“

\begin{tabular}{cc}
\hline $\begin{array}{c}\text { Gefahrene Geschwindigkeiten der } \\
\text { Kraftfahrzeuge (v85) }\end{array}$ & $\begin{array}{c}\text { Verträglichkeitsstufen } \\
\text { (mit den Anliegeransprüchen und dem Umfeld) }\end{array}$ \\
\hline$<30 \mathrm{~km} / \mathrm{h}$ & ++ (gut verträglich) \\
über 30 bis $35 \mathrm{~km} / \mathrm{h}$ & + (verträglich) \\
über 35 bis $40 \mathrm{~km} / \mathrm{h}$ & o (gerade noch verträglich) \\
über 40 bis $50 \mathrm{~km} / \mathrm{h}$ & - (unverträglich) \\
über $50 \mathrm{~km} / \mathrm{h}$ & -- (völlig unverträglich) \\
\hline
\end{tabular}

Quelle: eigene Darstellung nach Mörner et al. (1984)

1 Die v85 ist jene Geschwindigkeit, die von 85 Prozent der Fahrzeuge nicht überschritten wird. 


\section{e) Schwerverkehr/Lkw-Anteil}

Das Bewertungskriterium „Schwerverkehr/Lkw-Anteil“ bewertet den Lkw-Anteil am Kfz-Verkehrsaufkommen in der Normalstunde. Es wird von der Annahme ausgegangen, dass Schwerverkehr bei insgesamt geringem Verkehrsaufkommen insbesondere durch Lärmemissionen, aber auch hinsichtlich der Verkehrssicherheit störend für Anliegerlnnen wirkt. Für die Bewertung wird der Lkw-Verkehrsanteil am gesamten Kfz-Verkehrsaufkommen herangezogen und infolge die in Tabelle 7 beschriebenen Verträglichkeitsstufen festgesetzt.

Tabelle 7: Verträglichkeitsstufen für das Kriterium „Schwerverkehr/Lkw-Anteil“

\begin{tabular}{cc}
\hline $\begin{array}{c}\text { Lkw-Anteil am gesamten } \\
\text { Kfz-Verkehrsaufkommen }\end{array}$ & $\begin{array}{c}\text { Verträglichkeitsstufen } \\
\text { (mit den Anliegeransprüchen und dem Umfeld) }\end{array}$ \\
\hline$<3 \%$ & ++ (gut verträglich) \\
über 3 bis $6 \%$ & + (verträglich) \\
über 6 bis $9 \%$ & o (gerade noch verträglich) \\
über 9 bis $12 \%$ & - (unverträglich) \\
über $12 \%$ & -- (völlig unverträglich) \\
\hline
\end{tabular}

Quelle: eigene Darstellung nach Mörner et al. (1984)

\section{f) Trennwirkung: Querungshilfen/Umwege für Fußverkehr}

Das Bewertungskriterium „Trennwirkung: Querungshilfen/Umwege für Fußverkehr“ bewertet die Anzahl der Querungshilfen im Verhältnis zur Länge des Straßenabschnitts. Es wird davon ausgegangen, dass je nach Bebauungsstruktur bzw. je nach den vorher definierten Kategorien zum Straßenumfeld eine angemessene Anzahl an Querungsstellen nötig ist, um das Querungsbedürfnis von FußgängerInnen möglichst gut und ohne längere Umwege zu gewährleisten. Um den finalen Indikatorwert zu erhalten, wird das Verhältnis der Anzahl der Querungshilfen zur Länge des Straßenabschnitts noch mit 100 multipliziert, wodurch beispielsweise ein Wert von 1,0 eine durchschnittliche Distanz von 100 Metern zwischen zwei Querungshilfen bedeutet (vgl. Häfliger et al. 2015: 80f.). Tabelle 8 gibt einen Überblick zu den jeweiligen Verträglichkeitsstufen je Straßenumfeld bzw. Bebauungsstruktur des Gebiets. Da eine Begegnungszone überall gequert werden kann, wird nach Häfliger et al. (2015) angenommen, dass diese mit dem Umfeld immer gut verträglich ist (vgl. ebd.: 81).

Tabelle 8: Verträglichkeitsstufen des Kriteriums „Trennwirkung: Querungshilfen/Umwege für Fußverkehr“

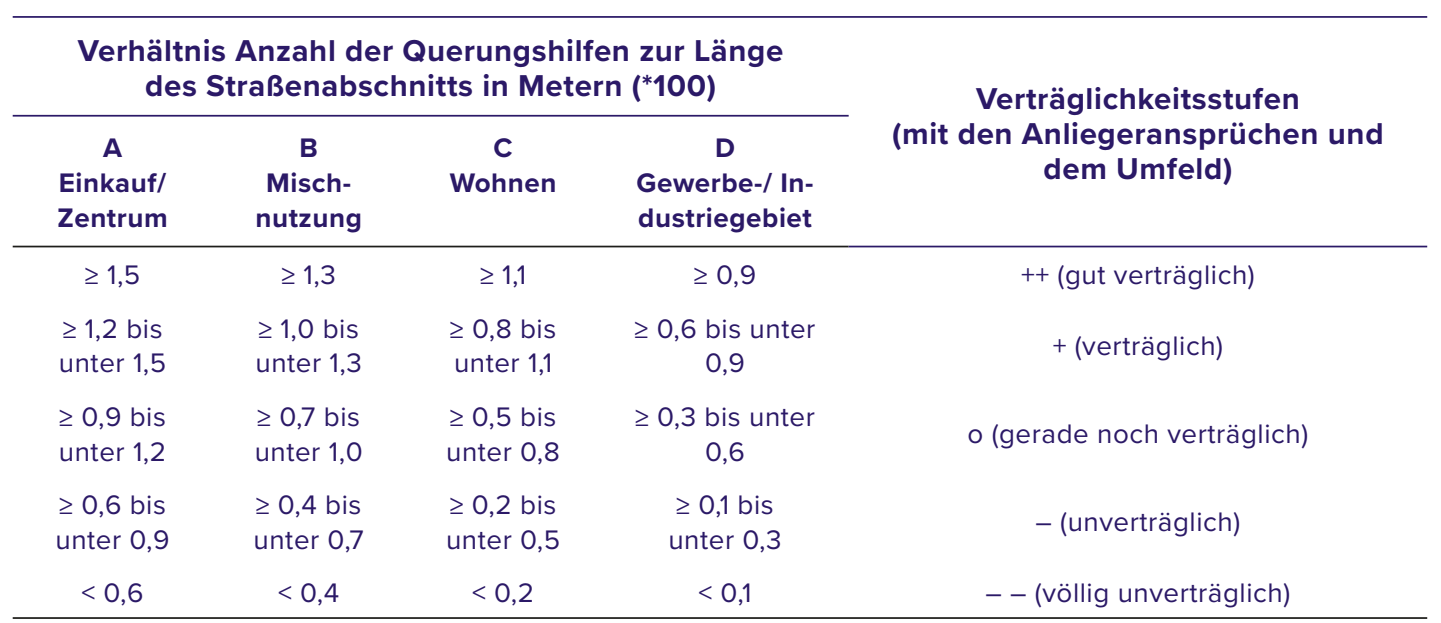




\subsubsection{Erhebung der erforderlichen Daten zur Bewertung der straßenräumlichen Verträglichkeit}

Für die oben beschriebene Vorgangsweise zur Bewertung der straßenräumlichen Verträglichkeit ist eine Bestimmung der relevanten Kriterien an den Straßenabschnitten vor Ort nötig. Zur Ermittlung der relevanten Daten für die Bewertung wurden eigene Erhebungen an allen untersuchten Straßenabschnitten durchgeführt. Hierbei wurden Verkehrszählungen zur Ermittlung der Anzahl der Fahrzeuge in der Spitzenstunde, der Anzahl von Fußgängerlnnen und Radfahrerlnnen sowie des Lkw-Anteils vorgenommen. Die Verkehrszählungen wurden im November 2019 an Werktagen jeweils in der Zeit zwischen 15 und 16 Uhr entsprechend den Empfehlungen für Verkehrserhebungen - EVE (vgl. FGSV 2012) an allen Straßenabschnitten durchgeführt. Die weiteren benötigten Daten zu den jeweiligen Kriterien wurden (1) (näherungsweise) aus der österreichischen Graphenintegrationsplattform ermittelt (Kriterium „gefahrene Geschwindigkeiten (v85)“) oder (2) vor Ort erhoben (Kriterien „Flächenaufteilung“, „Grün und Gestaltung“ und „Trennwirkung: Querungshilfen“).

\section{UNTERSUCHUNGSGEMEINDEN IN DER STADTREGION WIENS UND ANALYSIERTE STRASSENABSCHNITTE}

Abbildung 4: Übersicht der untersuchten Straßenabschnitte in den vier Untersuchungsgemeinden

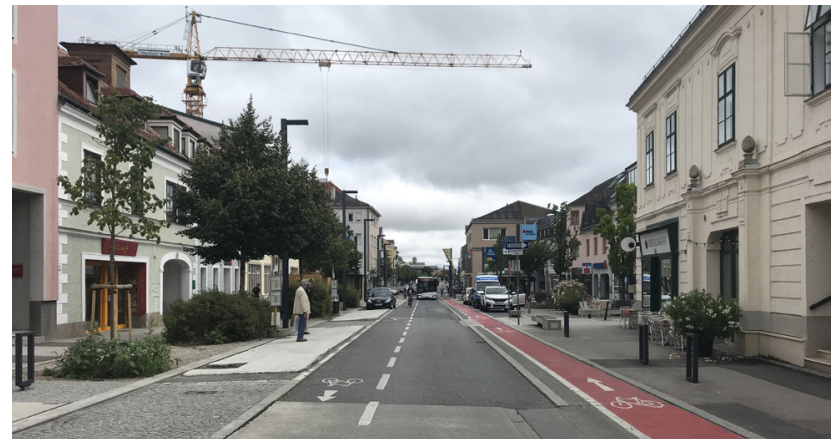

Hauptstraße, Mödling

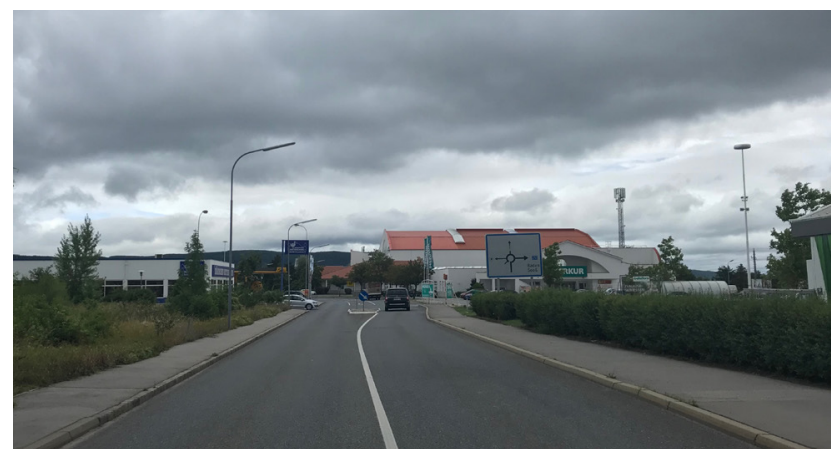

Industriestraße, Bad Vöslau

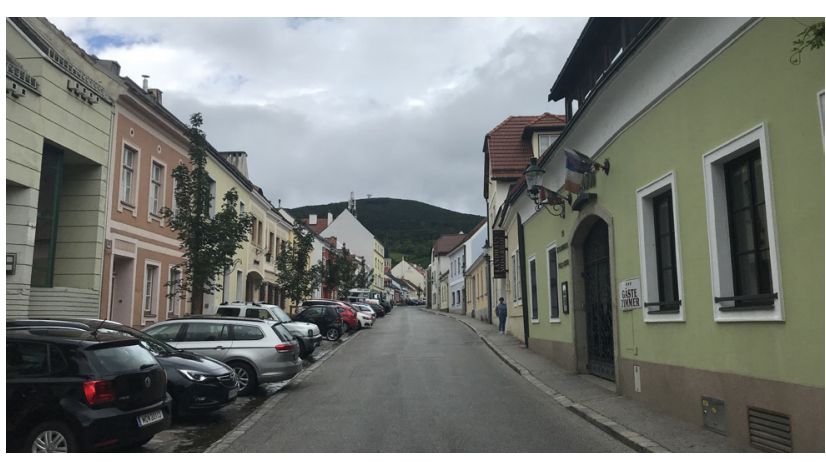

Wiener Straße, Gumpoldskirchen

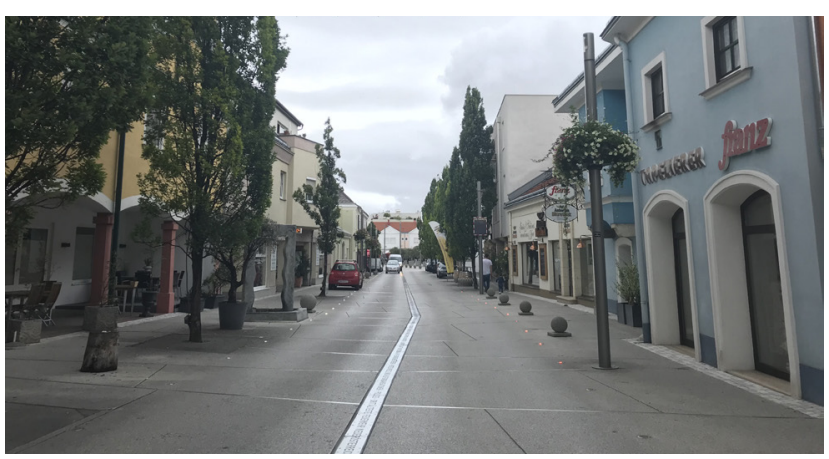

Hauptstraße, Leobersdorf

Quelle: Aggelos Soteropoulos 
Die Untersuchung der Automated Drivability sowie der straßenräumlichen Verträglichkeit wurde in den vier Beispielgemeinden

1. Mödling,

2. Gumpoldskirchen,

3. Bad Vöslau und

4. Leobersdorf

im Süden der Stadtregion Wien durchgeführt. Diese vier Gemeinden stehen stellvertretend für suburbane, eher ländlich-geprägte Gemeinden in der Stadtregion Wien. Abbildung 5 zeigt die Lage der untersuchten Beispielgemeinden in der Stadtregion Wien.

Abbildung 5: Lage der Untersuchungsgemeinden

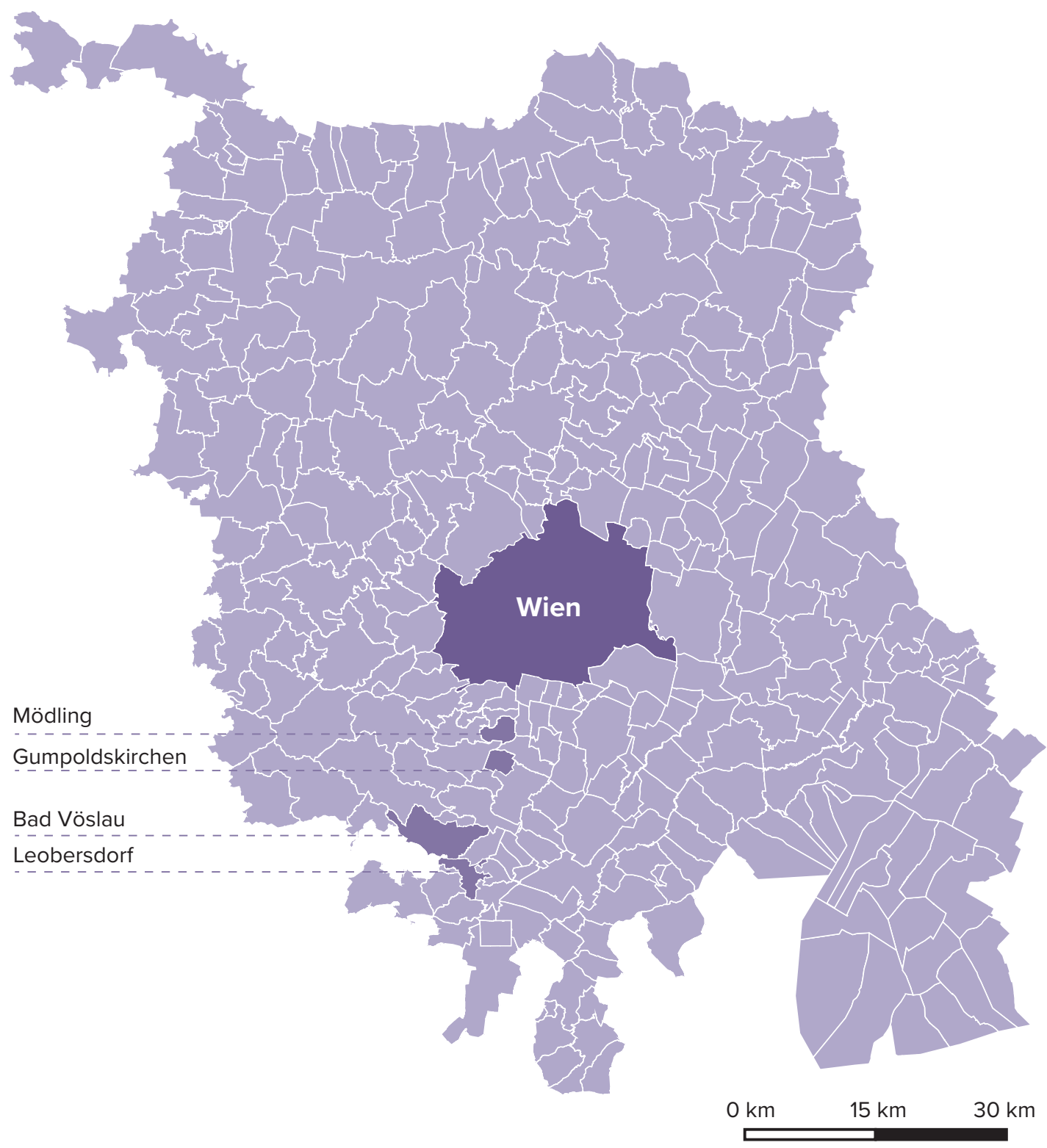

Quelle: eigene Darstellung 
Die Untersuchung der Automated Drivability fand im gesamten für den motorisierten Verkehr zugelassenen Straßennetz des Gemeindegebiets der jeweiligen vier Untersuchungsgemeinden statt, wobei das Straßennetz in Straßenabschnitte mit einer Länge von maximal 100 Metern aufgeteilt wurde, die als räumliche Bezugseinheiten für die Darstellung der Automated Drivability am geeignetsten erschienen (vgl. Su et al. 2019: 66).

Die Bewertung der straßenräumlichen Verträglichkeit wurde beispielhaft für jeweils eine StraBe bzw. einen Straßenabschnitt in den vier Untersuchungsgemeinden vorgenommen, darunter drei Ortsdurchfahrten und eine Gewerbestraße. Die Auswahl für diese vier Straßen erfolgte dabei einerseits aufgrund der Bedeutung dieser Straßentypen im suburbanen, zum Teil ländlich geprägten Raum sowie um andererseits auch die Heterogenität von Straßenräumen in diesen Gemeinden abzubilden bzw. zu berücksichtigen. Abbildung 4 zeigt einen Überblick der untersuchten Straßenabschnitte.

\section{ERGEBNISSE DER UNTERSUCHUNG}

Im Folgenden werden die Untersuchungsergebnisse in Bezug auf die Eignung der Straßenräume für den Einsatz automatisierter Fahrzeuge (Automated Drivability) und die straßenräumliche Verträglichkeit erläutert sowie eine Zusammenschau beider Aspekte vorgenommen.

\subsection{ERGEBNISSE ZUR EIGNUNG VON STRASSENRÄUMEN FÜR DEN EINSATZ AUTOMATISIERTER FAHRZEUGE (AUTOMATED DRIVABILITY)}

Abbildung 6 gibt einen Überblick zur Bewertung der Eignung von Straßenräumen für automatisierte Fahrzeuge, d. h. der Automated Drivability, in den vier Beispielgemeinden:

1. Mödling,

2. Gumpoldskirchen,

3. Bad Vöslau und

4. Leobersdorf.

Hohe Werte für die Automated Drivability und damit eine hohe Eignung für automatisierte Fahrzeuge zeigen sich in den Untersuchungsgemeinden insbesondere für Autobahnen, die im Osten von Bad Vöslau sowie westlich des Ortskerns von Leobersdorf erkennbar sind. Überdies sind hohe Werte für die Automated Drivability meist ebenso in jenen Teilen der Gemeinden erkennbar, welche sich in Anbindung an die Autobahnzu-/abfahrt befinden, so etwa nördlich des Ortskerns in Bad Vöslau oder westlich der Autobahn bzw. des Ortskerns in Leobersdorf. In Mödling sind hohe Werte der Automated Drivability nahezu ausschließlich im Gebiet um die Eisenbahnachse, in Gumpoldskirchen allenfalls im westlichen Teil der Gemeinde zu verzeichnen.

In den Ortskernen sowie auf einigen Zu-/Ausfahrtsstraßen der Untersuchungsgemeinden finden sich hingegen eher geringere Werte für die Automated Drivability. In Bad Vöslau sind 
hierbei insbesondere für die parallel zur B212 vom Ortskern nach Norden verlaufende Straße niedrige Werte der Automated Drivability erkennbar, in Mödling im Speziellen für die Straßen im Ortskern bzw. die Straßenabschnitte an der Kreuzung B11 (Spitalmühlgasse) und Neusiedler Straße. In Gumpoldskirchen sind vor allem für die Straßenabschnitte rund um den Kreisverkehr im westlichen Teil der Gemeinde niedrige Werte der Automated Drivability erkennbar, in Leobersdorf zeigt sich diese Gegebenheit im gesamten Ortskern sowie im Speziellen für die quer durch den Ortskern verlaufende Hauptstraße.

Abbildung 6: Automated Drivability in den vier Untersuchungsgemeinden

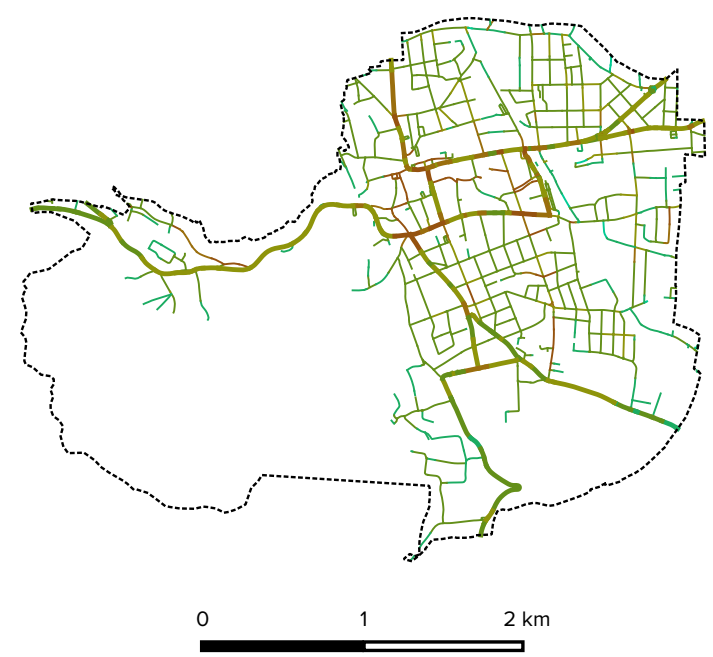

Mödling
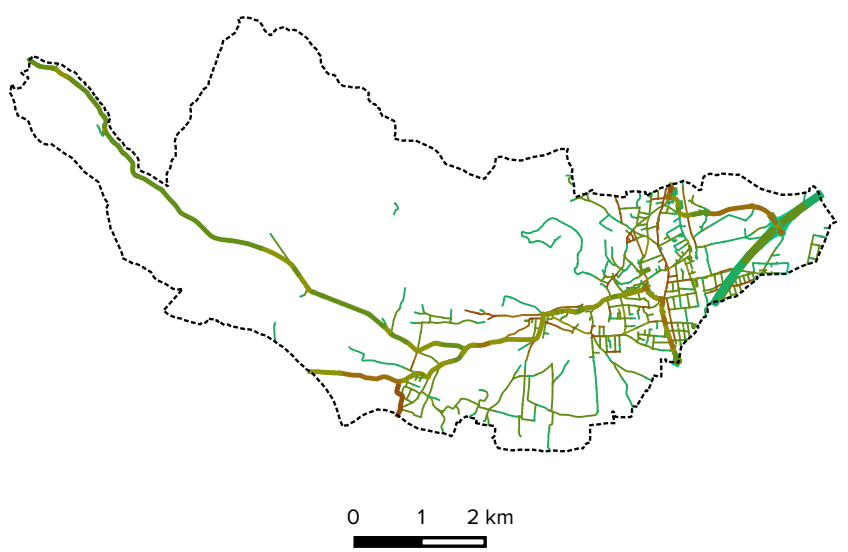

Bad Vöslau
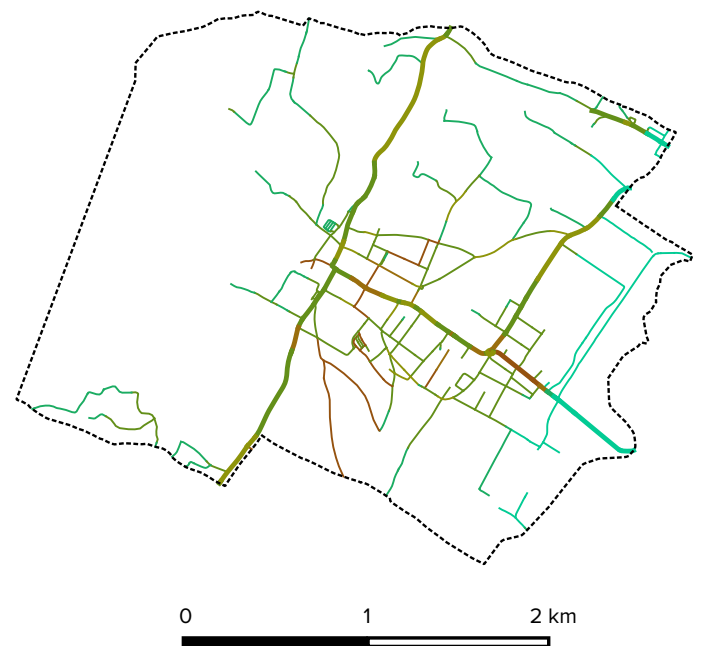

Gumpoldskirchen
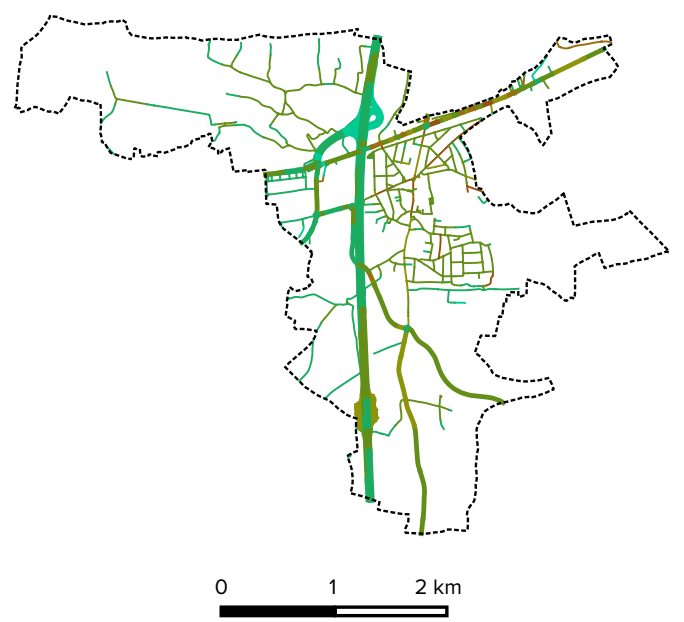

Leobersdorf geringe Eignung

(nur mit größeren Anpassungen) hohe Eignung

(ohne größere Anpassungen) 


\subsection{ERGEBNISSE ZUR STRASSENRÄUMLICHEN VERTRÄGLICHKEIT}

Im Folgenden wird die straßenräumliche Verträglichkeit in den vier Untersuchungsgemeinden Mödling, Gumpoldskirchen, Bad Vöslau und Leobersdorf analysiert.

\subsubsection{Grundwert der noch verträglichen Verkehrsbelastung und tatsächliche Verkehrshelastung}

Tabelle 9: Überblick zur Einordnung in das Straßenumfeld sowie den Grundwert der noch verträglichen Verkehrsbelastung bzw. Verträglichkeitsstufen für die untersuchten Straßenabschnitte

\begin{tabular}{ccc}
\hline Straßenabschnitt, Gemeinde & $\begin{array}{c}\text { Einordnung } \\
\text { Straßenumfeld }\end{array}$ & $\begin{array}{c}\text { Grundwert der noch verträglichen } \\
\text { Verkehrsbelastung bzW. } \\
\text { Verträglichkeitsstufen (Fz/Sp-h) }\end{array}$ \\
\hline Hauptstraße, Mödling & $\begin{array}{c}\text { < } 75 \text { (++ gut verträglich) } \\
\mathbf{7 5} \text { bis 150 (+ verträglich) } \\
\text { Kategorie A } \\
\text { Einkauf/Zentrum }\end{array}$ & $\begin{array}{c}>150 \text { bis 250 (o gerade noch verträglich) } \\
>250 \text { bis 400 (- unverträglich) } \\
>400 \text { (- - völlig unverträglich) }\end{array}$ \\
\hline
\end{tabular}

Wiener Straße, Gumpoldskirchen

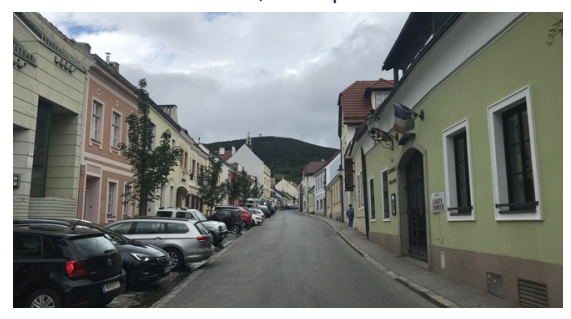

$<150$ (++ gut verträglich) 150 bis 250 (+ verträglich)

Kategorie B

Mischnutzung
$>250$ bis 400 (o gerade noch verträglich)

$>400$ bis 1.000 (- unverträglich)

$>1.000$ (- - völlig unverträglich)

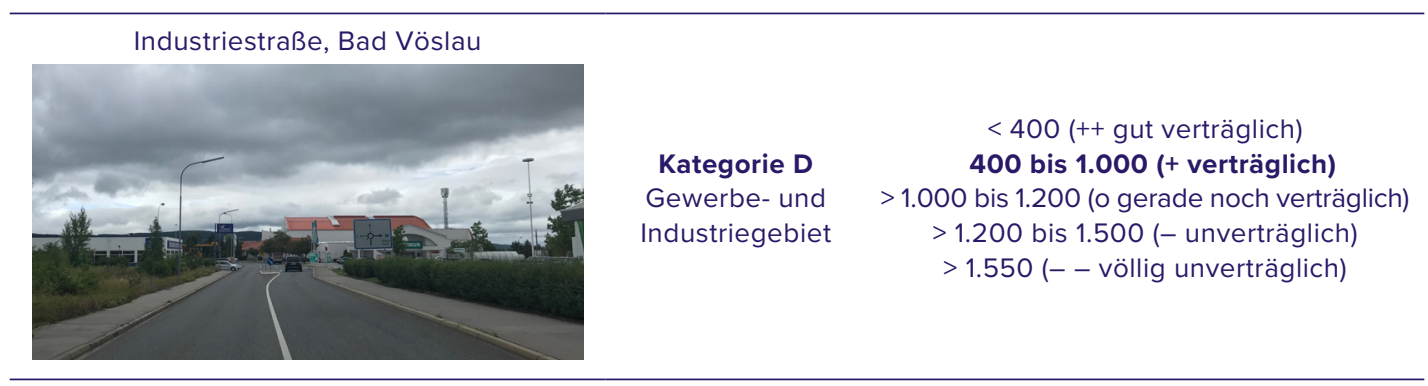

Hauptstraße, Leobersdorf

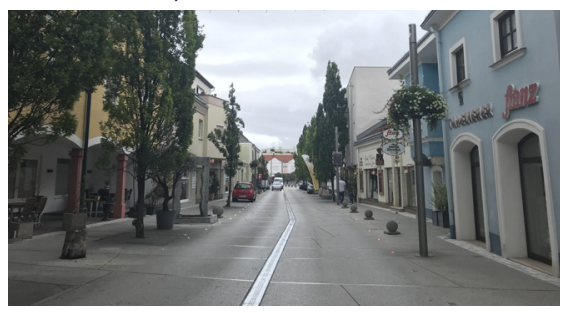

Kategorie A Einkauf/Zentrum
$<75$ (++ gut verträglich)

75 bis 150 (+ verträglich)

$>150$ bis 250 (o gerade noch verträglich)

$>250$ bis 400 (- unverträglich)

$>400$ (- - völlig unverträglich)

Quelle: eigene Darstellung nach Mörner et al. (1984) und Bühlmann/Laube (2013) 
Tabelle 9 zeigt die Einordnung des Straßenumfelds der vier untersuchten Straßenabschnitte in die vorher beschriebenen Kategorien sowie die dazugehörigen Grundwerte der noch verträglichen Verkehrsbelastung. Die Straßenabschnitte in den Gemeinden Mödling und Leobersdorf wurden hierbei der Kategorie „A: Einkauf/Zentrum“ mit einem Grundwert der noch verträglichen Verkehrsbelastung von $150 \mathrm{Fz} / \mathrm{Sp}$-h zugeordnet. Der Straßenabschnitt in Gumpoldskirchen lässt sich hingegen am ehesten der Kategorie „B: Mischnutzung“ zuteilen und weist somit einen Grundwert der noch verträglichen Verkehrsbelastung von $250 \mathrm{Fz} / \mathrm{Sp}-\mathrm{h}$ auf. Der Straßenabschnitt in Bad Vöslau wurde der Kategorie „D: Gewerbe- und Industriegebiet“ zugeordnet und besitzt somit einen Grundwert der noch verträglichen Verkehrsbelastung von 1.000 Fahrzeugen in der Spitzenstunde.

\subsubsection{Bestimmung der tatsächlichen noch verträglichen Verkehrsbelastung: Adap- tierung des Grundwerts durch Nutzung und Gestaltung des Straßenraums}

\section{a) Nutzung durch FußgängerInnen und RadfahrerInnen}

Im Hinblick auf das Kriterium „Nutzung durch FußgängerInnen (FG) und Radfahrerlnnen (RF)“ wurden sowohl für den Straßenabschnitt in Gumpoldskirchen (94 FG bzw. RF/Sp-h) als auch für jenen in Bad Vöslau (20 FG bzw. RF/Sp-h) unter 100 Fußgängerlnnen bzw. Radfahrerlnnen in der Spitzenstunde gezählt. Daraus folgt die Einstufung, dass der Kfz-Verkehr mit dieser Anzahl von FußgängerInnen bzw. Radfahrerlnnen gut verträglich ist und zu einer Adaptierung des Grundwerts der noch verträglichen Verkehrsbelastung um +50 Fz/Sp-h führt.

Am Straßenabschnitt in Leobersdorf wurden 196 FußgängerInnen bzw. Radfahrerlnnen pro Spitzenstunde erfasst, was den Kfz-Verkehr mit dieser Anzahl von FußgängerInnen bzw. RadfahrerInnen als verträglich einstufen lässt und zu einer Adaptierung des Grundwerts der noch verträglichen Verkehrsbelastung um $+25 \mathrm{Fz} / \mathrm{Sp}$-h führt. Mit der auf dem Straßenabschnitt in Mödling erhobenen Anzahl an Fußgängerlnnen und RadfahrerInnen (504 FG bzw. RG/Sp-h) hingegen ist der Kfz-Verkehr unverträglich und führt zu einer Adaptierung des Grundwerts der noch verträglichen Verkehrsbelastung um $-25 \mathrm{Fz} / \mathrm{Sp}-\mathrm{h}$.

Tabelle 10: Einstufung und Adaptierung des Grundwerts der noch verträglichen Verkehrsbelastung für das Kriterium „Nutzung durch FußgängerInnen und Radfahrerlnnen“

\begin{tabular}{|c|c|c|c|}
\hline $\begin{array}{c}\text { Straßenabschnitt, } \\
\text { Gemeinde }\end{array}$ & $\begin{array}{c}\text { Anzahl von } \\
\text { Fußgängerlnnen und } \\
\text { Radfahrerlnnen auf } \\
\text { der Straße in der } \\
\text { Spitzenstunde }\end{array}$ & $\begin{array}{l}\text { Verträglichkeits- } \\
\text { stufen (Verträglich- } \\
\text { keit des Kfz-Verkehrs } \\
\text { mit dieser Anzahl von } \\
\text { FußgängerInnen und } \\
\text { RadfahrerInnen) }\end{array}$ & $\begin{array}{l}\text { Adaptierung des } \\
\text { Grundwerts der noch } \\
\text { verträglichen } \\
\text { Verkehrsbelastung } \\
\text { (Fz/Sp-h) um }\end{array}$ \\
\hline $\begin{array}{l}\text { Hauptstraße, } \\
\text { Mödling }\end{array}$ & 504 & - (unverträglich) & -25 \\
\hline $\begin{array}{l}\text { Wiener Straße, } \\
\text { Gumpoldskirchen }\end{array}$ & 94 & ++ (gut verträglich) & +50 \\
\hline $\begin{array}{c}\text { Industriestraße, } \\
\text { Bad Vöslau }\end{array}$ & 20 & ++ (gut verträglich) & +50 \\
\hline $\begin{array}{l}\text { Hauptstraße, } \\
\text { Leobersdorf }\end{array}$ & 196 & + (verträglich) & +25 \\
\hline
\end{tabular}




\section{b) Flächenaufteilung}

Bezüglich des Kriteriums „Flächenaufteilung“, d. h. dem Verhältnis der Breite von Flächen für Fußgängerlnnen und Radfahrerlnnen zur Breite der Kfz-Verkehrsflächen, zeigen sich zwischen den einzelnen Straßenabschnitten zum Teil deutliche Unterschiede. Abbildung 7 zeigt eine Darstellung der Querprofile der vier Straßenabschnitte.

Abbildung 7: Straßenquerprofile der vier Straßenabschnitte in den Untersuchungsgemeinden

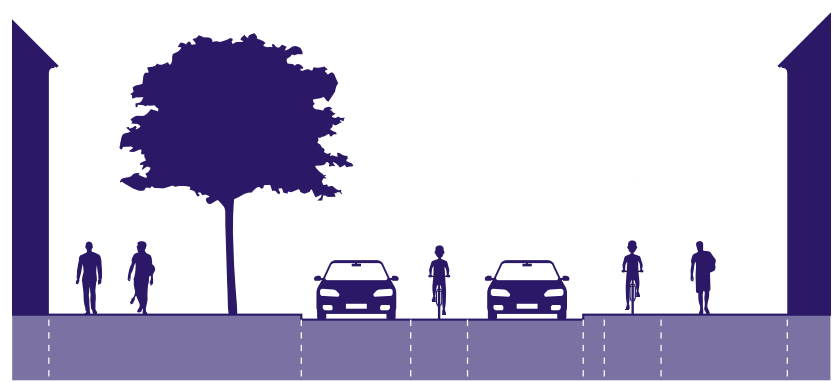

Hauptstraße, Mödling

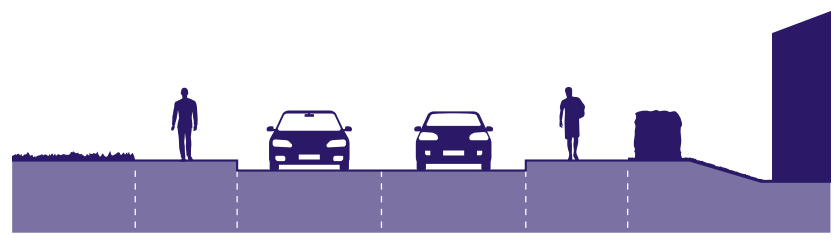

Industriestraße, Bad Vöslau

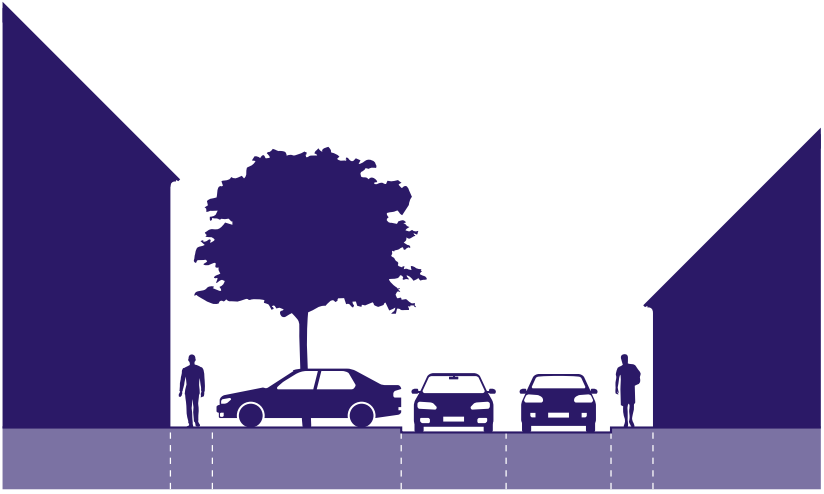

Wiener Straße, Gumpoldskirchen

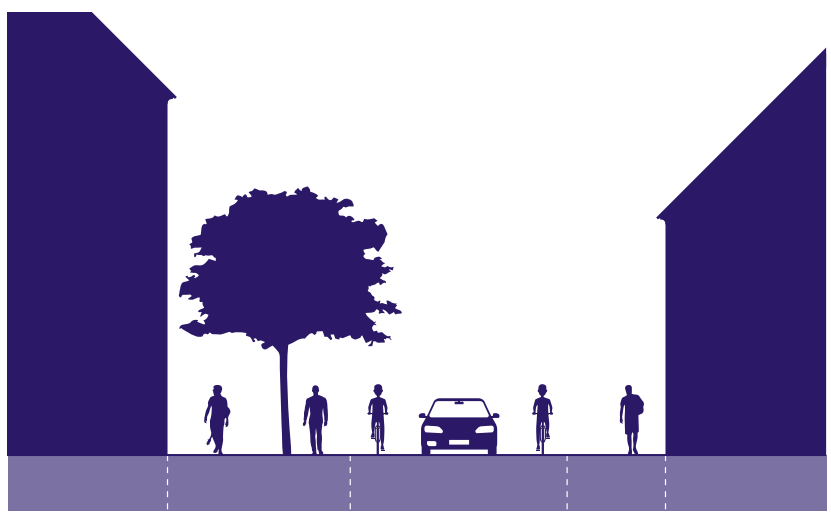

Hauptstraße, Leobersdorf

Quelle: eigene Darstellung

Für den Straßenabschnitt in Gumpoldskirchen zeigt sich für das Verhältnis der Breite von Flächen für Fußgängerlnnen und Radfahrerlnnen zur Breite der Kfz-Verkehrsflächen mit 0,18 ein sehr geringer Wert, was zu einer völlig unverträglichen Einstufung mit den Anliegeransprüchen und mit dem Umfeld und zu einer Adaptierung des Grundwerts der noch verträglichen Verkehrsbelastung von $-50 \mathrm{Fz} / \mathrm{Sp}-\mathrm{h}$ führt. Auch für den Straßenabschnitt in Bad Vöslau zeigt sich mit 0,57 ein eher geringer Wert, was einer gerade noch verträglichen Einstufung mit den Anliegeransprüchen und mit dem Umfeld und einer Adaptierung des Grundwerts der noch verträglichen Verkehrsbelastung von $\pm 0 \mathrm{Fz} / \mathrm{Sp}-\mathrm{h}$ zuzuordnen ist.

Für die Straßenabschnitte in Mödling und Leobersdorf zeigen sich mit Werten von 1,17 und 0,75 hingegen positiver zu bewertende Verhältnisse der Breite von Flächen für Fußgängerlnnen und RadfahrerInnen zur Breite der Kfz-Verkehrsflächen, die mit den Anliegeransprüchen verträglich bzw. gut verträglich sind. Dies führt zur Adaptierung des Grundwerts der noch verträglichen Verkehrsbelastung um +50 bzw. +25 Fz Sp-h. 
Tabelle 11: Einstufung und Adaptierung des Grundwerts der noch verträglichen Verkehrsbelastung für das Kriterium „Flächenaufteilung“

\begin{tabular}{|c|c|c|c|}
\hline $\begin{array}{c}\text { Straßenabschnitt, } \\
\text { Gemeinde }\end{array}$ & $\begin{array}{l}\text { Verhältnis der Breite } \\
\text { von Flächen für } \\
\text { Fußgängerlnnen und } \\
\text { Radfahrerlnnen zur } \\
\text { Breite der Kfz-Ver- } \\
\text { kehrsflächen }\end{array}$ & $\begin{array}{l}\text { Verträglichkeitsstufen } \\
\text { (mit den Anlieger- } \\
\text { ansprüchen und mit } \\
\text { dem Umfeld) }\end{array}$ & $\begin{array}{c}\text { Adaptierung des } \\
\text { Grundwerts der noch } \\
\text { verträglichen } \\
\text { Verkehrsbelastung } \\
\text { (Fz/Sp-h) um }\end{array}$ \\
\hline $\begin{array}{l}\text { Hauptstraße, } \\
\text { Mödling }\end{array}$ & 1,17 & ++ (gut verträglich) & +50 \\
\hline $\begin{array}{l}\text { Wiener Straße, } \\
\text { Gumpoldskirchen }\end{array}$ & 0,18 & - - (völlig unverträglich) & -50 \\
\hline $\begin{array}{c}\text { Industriestraße, } \\
\text { Bad Vöslau }\end{array}$ & 0,57 & $\begin{array}{l}\text { o (gerade noch } \\
\text { verträglich) }\end{array}$ & \pm 0 \\
\hline $\begin{array}{l}\text { Hauptstraße, } \\
\text { Leobersdorf }\end{array}$ & 0,75 & + (verträglich) & +25 \\
\hline
\end{tabular}

Quelle: eigene Darstellung

\section{c) Grün und Gestaltung}

Hinsichtlich des Kriteriums „Grün und Gestaltung“ zeigt sich bei den Straßenabschnitten in Mödling und Leobersdorf, dass dort Grün und sonstige gestalterische Elemente eindeutig den Straßenraum prägen bzw. objektiv wahrnehmbar sind und dies mit den Anliegeransprüchen als gut verträglich bzw. verträglich eingeordnet werden kann. Dies führt zu einer Adaptierung des Grundwerts der noch verträglichen Verkehrsbelastung um +25 bzw. +50 Fz/Sp-h. Für den StraBenabschnitt in Gumpoldskirchen wird hingegen erkennbar, dass sich Grün und gestalterische Elemente in ihrer Wirkung gegen ungestaltete Flächen eher aufheben, was mit den Anliegeransprüchen und mit dem Umfeld nur gerade noch verträglich ist (Adaptierung des Grundwerts der noch verträglichen Verkehrsbelastung um \pm 0 Fz/Sp-h). Auf dem Straßenabschnitt in Bad Vöslau fehlen Grün und sonstige gestalterische Elemente ganz, was mit den Anliegeransprüchen und mit dem Umfeld völlig unverträglich ist und zu einer Adaptierung des Grundwerts der noch verträglichen Verkehrsbelastung um -50 Fz/Sp-h führt.

\section{d) Geschwindigkeit}

Bezüglich des Kriteriums „Geschwindigkeit“ zeigen sich in den vier Straßenabschnitten laut GIP unterschiedliche gefahrene Geschwindigkeiten der Kraftfahrzeuge (v85) ${ }^{2}$. Mit 35 km/h bzw. 20 $\mathrm{km} / \mathrm{h}$ finden sich auf dem Straßenabschnitt in Gumpoldskirchen und Leobersdorf relativ geringe gefahrene Geschwindigkeiten, die mit den Anliegeransprüchen und mit dem Umfeld gut verträglich bzw. verträglich sind und zu einer Adaptierung des Grundwerts der noch verträglichen Verkehrsbelastung um +25 bzw. +50 Fz/Sp-h führen. Auf dem Straßenabschnitt in Mödling zeigt sich eine gefahrene Geschwindigkeit der Kraftfahrzeuge (v85) von 40 km/h, die mit den Anliegeransprüchen und mit dem Umfeld nur gerade noch verträglich ist (Adaptierung des Grundwerts der noch verträglichen Verkehrsbelastung um $\pm 0 \mathrm{Fz} / \mathrm{Sp}-\mathrm{h})$. Die gefahrenen Geschwindigkeiten der Kraftfahrzeuge (v85) auf dem Straßenabschnitt in Bad Vöslau betragen $50 \mathrm{~km} / \mathrm{h}$ und sind damit mit den Anliegeransprüchen und dem Umfeld unverträglich, was zu einer Adaptierung des Grundwerts der noch verträglichen Verkehrsbelastung um -25 Fz/Sp-h führt.

2 Die in der GIP enthaltenen v85-Geschwindigkeiten stammen im Idealfall aus Messungen. Sofern im Rahmen der Datenübermittlung zur GIP keine zuverlässigeren Geschwindigkeiten bekannt sind, werden in der GIP je nach Straßenkategorien und unterschieden nach Freiland und Ortsgebiet Standardwerte (aus empirischen Untersuchungen) zur Eintragung vorgegeben (vgl. GIP.at 2019: 123). 
Tabelle 12: Einstufung und Adaptierung des Grundwerts der noch verträglichen Verkehrsbelastung für das Kriterium „Grün und Gestaltung“

\begin{tabular}{|c|c|c|c|}
\hline $\begin{array}{l}\text { Straßenab- } \\
\text { schnitt, } \\
\text { Gemeinde }\end{array}$ & $\begin{array}{c}\text { Grün und Gestaltung des } \\
\text { Straßenraums }\end{array}$ & $\begin{array}{c}\text { Verträglichkeitsstufen } \\
\text { (mit den Anlieger- } \\
\text { ansprüchen und dem } \\
\text { Umfeld) }\end{array}$ & $\begin{array}{l}\text { Adaptierung des } \\
\text { Grundwerts der } \\
\text { noch verträglichen } \\
\text { Verkehrsbelastung } \\
\text { (Fz/Sp-h) um }\end{array}$ \\
\hline $\begin{array}{l}\text { Hauptstraße, } \\
\text { Mödling }\end{array}$ & $\begin{array}{l}\text { Grün und sonstige gestalterische } \\
\text { Elemente sind objektiv } \\
\text { wahrnehmbar. Sie überwiegen } \\
\text { insgesamt gesehen im } \\
\text { Erscheinungsbild der Straße } \\
\text { noch gegenüber der technischen } \\
\text { Verkehrsanlage. }\end{array}$ & $\begin{array}{c}+ \\
\text { (verträglich) }\end{array}$ & +25 \\
\hline $\begin{array}{l}\text { Wiener Straße, } \\
\text { Gumpoldskirchen }\end{array}$ & $\begin{array}{c}\text { Grün und gestalterische } \\
\text { Elemente heben sich in ihrer } \\
\text { Wirkung gegen ungestaltete } \\
\text { Flächen auf, Grün und } \\
\text { Gestaltung prägen die Straße } \\
\text { nicht. }\end{array}$ & $\begin{array}{c}\mathrm{O} \\
\text { (gerade noch verträglich) }\end{array}$ & \pm 0 \\
\hline $\begin{array}{l}\text { Industriestraße, } \\
\text { Bad Vöslau }\end{array}$ & $\begin{array}{l}\text { Grün und sonstige gestalterische } \\
\text { Elemente fehlen ganz, der } \\
\text { Straßenraum ist kahl und nackt. }\end{array}$ & $\begin{array}{c}-- \\
\text { (völlig unverträglich) }\end{array}$ & -50 \\
\hline $\begin{array}{l}\text { Hauptstraße, } \\
\text { Leobersdorf }\end{array}$ & $\begin{array}{c}\text { Grün und sonstige gestalterische } \\
\text { Elemente bestimmen eindeutig } \\
\text { den Straßenraum und } \\
\text { prägen den Charakter der } \\
\text { Straße. Sie führen zu einer } \\
\text { Unverwechselbarkeit der Straße } \\
\text { mit hohem Erlebniswert. }\end{array}$ & $\begin{array}{c}++ \\
\text { (gut verträglich) }\end{array}$ & +50 \\
\hline
\end{tabular}

Quelle: eigene Darstellung

Tabelle 13: Einstufung und Adaptierung des Grundwerts der noch verträglichen Verkehrsbelastung für das Kriterium „Geschwindigkeit“

\begin{tabular}{cccc}
\hline $\begin{array}{c}\text { Straßenabschnitt, } \\
\text { Gemeinde }\end{array}$ & $\begin{array}{c}\text { Gefahrene Geschwin- } \\
\text { digkeiten der } \\
\text { Kraftfahrzeuge (v85) }\end{array}$ & $\begin{array}{c}\text { Verträglichkeitsstufen } \\
\text { (mit den Anlieger- } \\
\text { ansprüchen und dem } \\
\text { Umfeld) }\end{array}$ & $\begin{array}{c}\text { Adaptierung des } \\
\text { Grundwerts der noch } \\
\text { verträglichen } \\
\text { Verkehrsbelastung } \\
\text { (Fz/Sp-h) um }\end{array}$ \\
\hline $\begin{array}{c}\text { Hauptstraße, } \\
\text { Mödling }\end{array}$ & $40 \mathrm{~km} / \mathrm{h}$ & $\begin{array}{c}\text { o (gerade noch } \\
\text { verträglich) }\end{array}$ & \pm 0 \\
$\begin{array}{c}\text { Wiener Straße, } \\
\text { Gumpoldskirchen } \\
\text { Industriestraße, } \\
\text { Bad Vöslau }\end{array}$ & $35 \mathrm{~km} / \mathrm{h}$ & + (verträglich) & +25 \\
$\begin{array}{c}\text { Hauptstraße, } \\
\text { Leobersdorf }\end{array}$ & $50 \mathrm{~km} / \mathrm{h}$ & - (unverträglich) & -25 \\
\end{tabular}




\section{e) Schwerverkehr/Lkw-Anteil}

Hinsichtlich des Kriteriums „Schwerverkehr/Lkw-Anteil“ zeigt sich auf dem Straßenabschnitt in Bad Vöslau mit 5 Prozent der höchste Lkw-Anteil am gesamten Kfz-Verkehrsaufkommen, was mit den Anliegeransprüchen und mit dem Umfeld verträglich ist und zu einer Adaptierung des Grundwerts der noch verträglichen Verkehrsbelastung um +25 Fz/Sp-h führt. Die gleiche Einstufung erfolgt auch für den Straßenabschnitt in Gumpoldskirchen, für welchen ein Lkw-Anteil von 4 Prozent erkennbar ist. Sowohl der Straßenabschnitt in Mödling als auch jener in Leobersdorf weist mit 1 bzw. 2 Prozent sehr geringe Lkw-Anteile auf, was mit den Anliegeransprüchen und mit dem Umfeld gut verträglich ist und zu einer Adaptierung des Grundwerts der noch verträglichen Verkehrsbelastung um $+50 \mathrm{Fz} / \mathrm{Sp}-\mathrm{h}$ führt.

Tabelle 14: Einstufung und Adaptierung des Grundwerts der noch verträglichen Verkehrsbelastung für das Kriterium „Schwerverkehr/Lkw-Anteil“

\begin{tabular}{|c|c|c|c|}
\hline $\begin{array}{c}\text { Straßenabschnitt, } \\
\text { Gemeinde }\end{array}$ & $\begin{array}{l}\text { Lkw-Anteil am } \\
\text { gesamten Kfz-Ver- } \\
\text { kehrsaufkommen }\end{array}$ & $\begin{array}{c}\text { Verträglichkeitsstufen } \\
\text { (mit den Anlieger- } \\
\text { ansprüchen und dem } \\
\text { Umfeld) }\end{array}$ & $\begin{array}{l}\text { Adaptierung des } \\
\text { Grundwerts der noch } \\
\text { verträglichen } \\
\text { Verkehrsbelastung } \\
\text { (Fz/Sp-h) um }\end{array}$ \\
\hline $\begin{array}{l}\text { Hauptstraße, } \\
\text { Mödling }\end{array}$ & $1 \%$ & ++ (gut verträglich) & +50 \\
\hline $\begin{array}{l}\text { Wiener Straße, } \\
\text { Gumpoldskirchen }\end{array}$ & $4 \%$ & + (verträglich) & +25 \\
\hline $\begin{array}{l}\text { Industriestraße, } \\
\text { Bad Vöslau }\end{array}$ & $5 \%$ & + (verträglich) & +25 \\
\hline $\begin{array}{l}\text { Hauptstraße, } \\
\text { Leobersdorf }\end{array}$ & $2 \%$ & ++ (gut verträglich) & +50 \\
\hline
\end{tabular}

Quelle: eigene Darstellung

f) Trennwirkung: Querungshilfen/Umwege für Fußverkehr

Hinsichtlich des Kriteriums „Trennwirkung: Querungshilfen/Umwege für Fußverkehr“ zeigt sich bei den Straßenabschnitten in Mödling und Gumpoldskirchen eine im Vergleich zur Länge

Tabelle 15: Einstufung und Adaptierung des Grundwerts der noch verträglichen Verkehrsbelastung für das Kriterium „Trennwirkung: Querungshilfen/Umwege für Fußverkehr“

\begin{tabular}{ccccc}
\hline $\begin{array}{c}\text { Straßenabschnitt, } \\
\text { Gemeinde }\end{array}$ & $\begin{array}{c}\text { Kategorie } \\
\text { Straßen- } \\
\text { umfeld }\end{array}$ & $\begin{array}{c}\text { Verhältnis der An- } \\
\text { zahl der Querungs- } \\
\text { hilfen zur Länge des } \\
\text { Straßenabschnitts } \\
(\mathbf{1 0 0})\end{array}$ & $\begin{array}{c}\text { Verträglichkeits- } \\
\text { stufen (mit den } \\
\text { Anliegeransprü- } \\
\text { chen und dem } \\
\text { Umfeld) }\end{array}$ & $\begin{array}{c}\text { Adaptierung des } \\
\text { Grundwerts der } \\
\text { noch verträglichen } \\
\text { Verkehrsbelastung } \\
\text { (Fz/Sp-h) um }\end{array}$ \\
\hline $\begin{array}{c}\text { Hauptstraße, } \\
\text { Mödling }\end{array}$ & A & 0,87 & - (unverträglich) & -25 \\
$\begin{array}{c}\text { Wiener Straße, } \\
\text { Gumpoldskirchen }\end{array}$ & B & 0,48 & - (unverträglich) & -25 \\
$\begin{array}{c}\text { Industriestraße, } \\
\text { Bad Vöslau }\end{array}$ & D & 0,58 & o (gerade noch \\
verträglich) & \pm 0 \\
$\begin{array}{c}\text { Hauptstraße, } \\
\text { Leobersdorf }\end{array}$ & A & Begegnungszone & ++ (gut verträglich) & +50 \\
\hline
\end{tabular}


des Straßenabschnitts und zum Straßenumfeld geringe Anzahl von Querungshilfen, die mit den Anliegeransprüchen und dem Umfeld unverträglich sind und jeweils zu einer Adaptierung des Grundwerts der noch verträglichen Verkehrsbelastung um -25 Fz/Sp-h führen. Beim Straßenabschnitt in Bad Vöslau ist die Anzahl der Querungshilfen im Vergleich zur Länge des Straßenabschnitts gerade noch mit den Anliegeransprüchen und dem Umfeld verträglich (Adaptierung des Grundwerts der noch verträglichen Verkehrsbelastung um $\pm 0 \mathrm{Fz} / \mathrm{Sp}$-h). Der Straßenabschnitt in Leobersdorf hingegen ist aufgrund der existierenden Begegnungszone hinsichtlich Querungshilfen mit den Anliegeransprüchen und dem Umfeld gut verträglich, was zu einer Adaptierung des Grundwerts der noch verträglichen Verkehrsbelastung um $+50 \mathrm{Fz} /$ Sp-h führt.

\subsubsection{Zusammenfassende Bewertung der straßenräumlichen Verträglichkeit}

Tabelle 16 (auf der folgenden Seite) gibt einen zusammenfassenden Überblick zu den

a. Grundwerten der noch verträglichen Verkehrsbelastung,

b. den adaptierten Gesamtwerten der verträglichen Verkehrsbelastung bzw. der Verträglichkeitsstufen und

c. zur tatsächlichen Verkehrsbelastung sowie

d. zur Einstufung der straßenräumlichen Verträglichkeit

für alle vier untersuchten Straßenabschnitte.

Außer für den Straßenabschnitt in Bad Vöslau (bei den Grundwerten der jeweiligen Verträglichkeitsstufen durch die Adaptierung ergibt sich keine Veränderung, der Grundwert der verträglichen Verkehrsbelastung liegt auch nach der Adaptierung bei $1.000 \mathrm{Fz} / \mathrm{Sp}-\mathrm{h}$ ) fällt die Adaptierung des Grundwerts der noch verträglichen Verkehrsbelastung in den anderen drei Gemeinden positiv aus.

Für den Straßenabschnitt in Mödling erhöhen sich die Grundwerte der jeweiligen Verträglichkeitsstufen um $75 \mathrm{Fz} / \mathrm{Sp}$-h, der adaptierte Grundwert der verträglichen Verkehrsbelastung liegt somit bei $225 \mathrm{Fz} / \mathrm{Sp}$-h, in Gumpoldskirchen erhöhen sich die Grundwerte der jeweiligen Verträglichkeitsstufen um $25 \mathrm{Fz} / \mathrm{Sp}$-h, der adaptierte Grundwert der verträglichen Verkehrsbelastung liegt somit bei $275 \mathrm{Fz} / \mathrm{Sp}$-h. In Leobersdorf erhöhen sich die Grundwerte der jeweiligen Verträglichkeitsstufen für den Straßenabschnitt um $250 \mathrm{Fz} / \mathrm{Sp}-\mathrm{h}$, wodurch sich ein adaptierter Grundwert der verträglichen Verkehrsbelastung von $400 \mathrm{Fz} / \mathrm{Sp}-\mathrm{h}$ ergibt .

Unter Berücksichtigung der tatsächlichen Verkehrsbelastung (Fz/Sp-h) in den einzelnen StraBenabschnitten und der adaptierten Verträglichkeitsstufen für die vier Straßenabschnitte zeigt sich somit, dass allein für den Straßenabschnitt in Gumpoldskirchen die tatsächliche Kfz-Verkehrsbelastung des Straßenabschnitts mit den Ansprüchen aus dem Straßenumfeld gut verträglich ist. Für den Straßenabschnitt in Bad Vöslau ist die tatsächliche Kfz-Verkehrsbelastung mit den Ansprüchen aus dem Straßenumfeld gerade noch verträglich. Hingegen ist die tatsächliche Kfz-Verkehrsbelastung auf den Straßenabschnitten in Mödling und Leobersdorf mit den Ansprüchen aus dem Straßenumfeld unverträglich. 


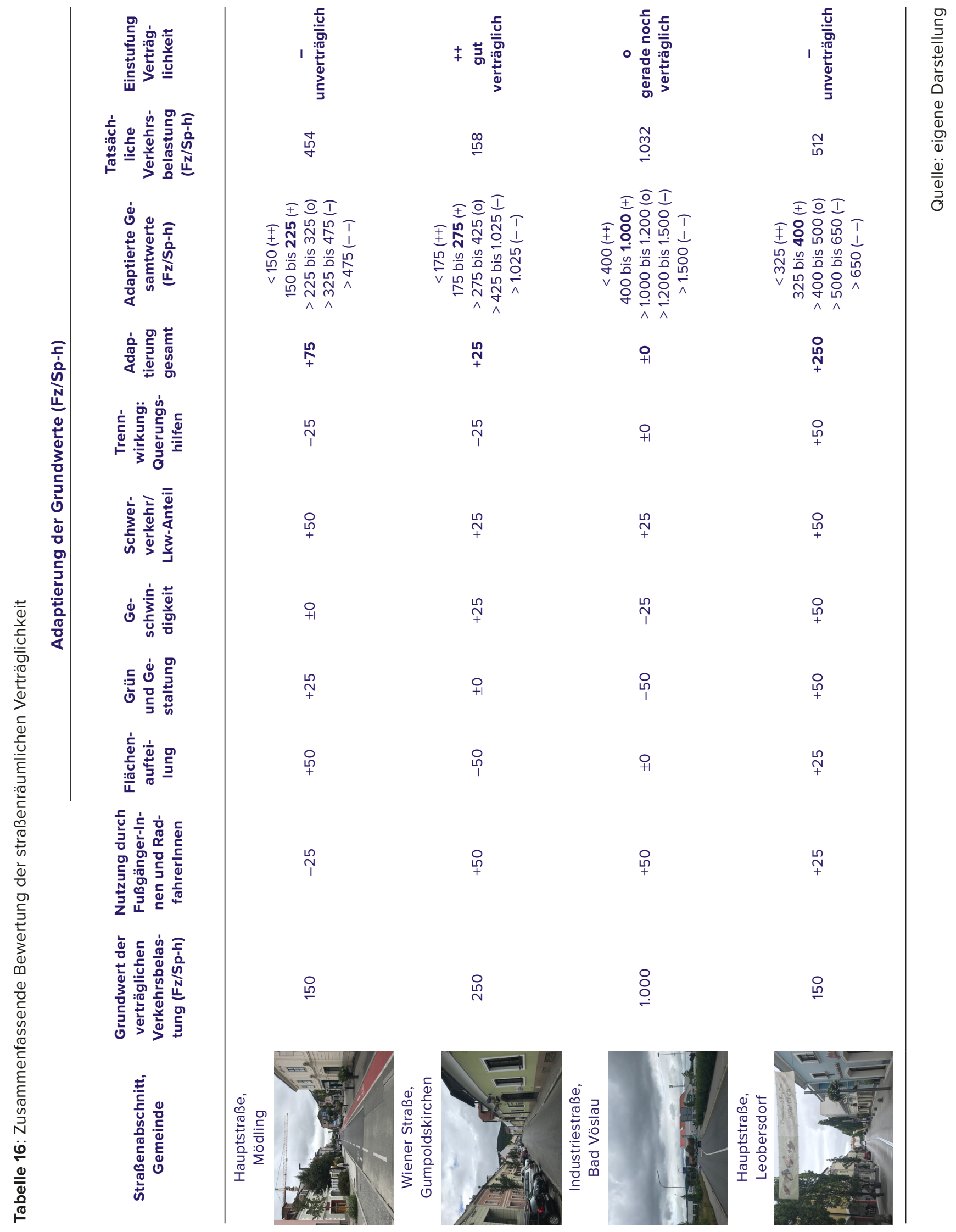




\subsection{ZUSAMMENSCHAU DER ERGEBNISSE ZUR AUTOMATED DRIVABILITY UND STRASSENRÄUMLICHEN VERTRÄGLICHKEIT}

Tabelle 17: Gegenüberstellung der Bewertung von Automated Drivability und straßenräumlicher Verträglichkeit für alle untersuchten Straßenabschnitte

\begin{tabular}{ccc}
$\begin{array}{c}\text { Straßenabschnitt, } \\
\text { Gemeinde }\end{array}$ & $\begin{array}{c}\text { Einstufung } \\
\text { Automated Drivability }\end{array}$ & $\begin{array}{c}\text { Einstufung } \\
\text { Verträglichkeit }\end{array}$ \\
\hline Hauptstraße, Mödling & eher gering $(0,641)$ & - unverträglich \\
\hline
\end{tabular}

Wiener Straße, Gumpoldskirchen

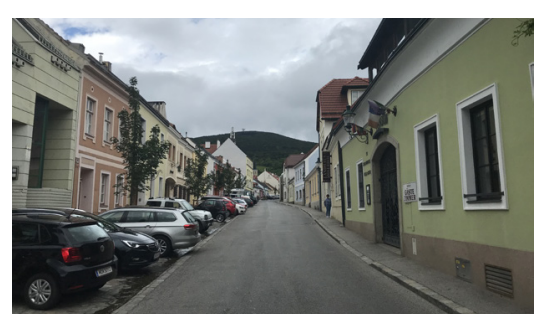

Industriestraße, Bad Vöslau

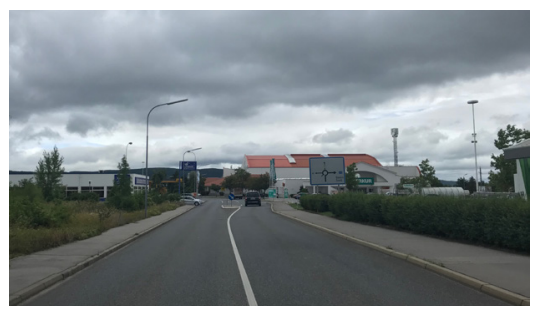

Hauptstraße, Leobersdorf

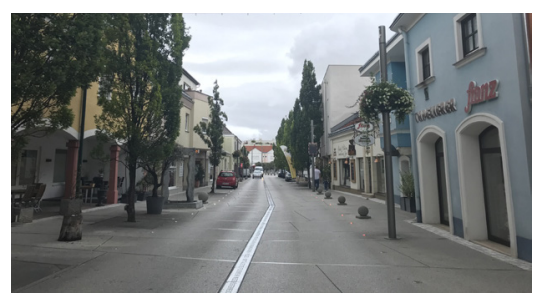


In Tabelle 17 wird die Bewertung der Straßenabschnitte hinsichtlich der straßenräumlichen Verträglichkeit jener der Automated Drivability, d. h. der Eignung für automatisierte Fahrzeuge, gegenübergestellt. In der Tendenz zeigt sich dabei, dass bei den Straßenabschnitten in Mödling und Leobersdorf, die eine eher geringe Einstufung der Automated Drivability aufweisen, die tatsächliche Kfz-Verkehrsbelastung mit den Ansprüchen des Straßenumfelds unverträglich ist. Beim Straßenabschnitt in Bad Vöslau, der eine eher hohe Eignung für automatisierte Fahrzeuge aufweist, ist die tatsächliche Kfz-Verkehrsbelastung mit den Ansprüchen des Straßenumfelds hingegen gerade noch verträglich. Beim Straßenabschnitt in Gumpoldskirchen, der eine geringe bis mittlere Eignung für automatisierte Fahrzeuge aufweist, ist die tatsächliche Kfz-Verkehrsbelastung mit den Ansprüchen des Straßenumfelds hingegen sogar gut verträglich.

\section{DISKUSSION UND FAZIT}

Im Rahmen des Beitrag erfolgte eine Untersuchung der Eignung von Straßenräumen für automatisierte Fahrzeuge (Automated Drivability) im Stadt-Land-Kontinuum am Beispiel von vier verschiedenen Gemeinden in der Stadtregion Wien. Zudem wurde an einzelnen beispielhaften Straßenabschnitten in den vier Untersuchungsgemeinden eine Analyse der straßenräumlichen Verträglichkeit durchgeführt, d. h. es wurde untersucht, inwieweit die derzeitige tatsächliche Kfz-Verkehrsbelastung an den Straßenabschnitten mit den Ansprüchen des Straßenumfelds verträglich ist. Anschließend wurden beide Bewertungen für die vier Straßenabschnitte einander gegenübergestellt.

Die Ergebnisse zeigen, dass sich in den oftmals historischen Ortskernen der Untersuchungsgemeinden eher geringe Werte der Automated Drivability zeigen und diese Straßen somit für den Einsatz automatisierter Fahrzeuge eher schlecht geeignet sind. Typischerweise handelt es sich hierbei um enge Straßen mit Geschäften und einem höheren Aufkommen an Fußgängerlnnen und Radfahrerlnnen, welche erschwerte Bedingungen für automatisierte Fahrzeuge aufweisen. Automatisierte Fahrzeuge sind hierbei allenfalls mit sehr geringen Geschwindigkeiten oder durch entsprechende Infrastrukturanpassungen (baulich, digital) denkbar. Besonders auf den Abschnitten der Autobahnen sowie in manchen Teilen der Gemeinden außerhalb des Ortskerns - und hier insbesondere in Gewerbe- oder Wohngebieten mit geringen Geschwindigkeitsniveaus - ist hingegen eine höhere Eignung für automatisierte Fahrzeuge erkennbar. Automatisierte Fahrzeuge könnten hier leichter und mit einer geringeren Notwendigkeit von Anpassungen unterwegs sein.

Hinsichtlich der straßenräumliche Verträglichkeit zeigen sich für die drei ausgewählten StraBenabschnitte von Ortsdurchfahrten sowie für den ausgewählten Straßenabschnitt im Industriegebiet sehr unterschiedliche Ergebnisse. Allein für die Ortsdurchfahrt in Gumpoldskirchen ist die derzeitige tatsächliche Kfz-Verkehrsbelastung der Straße mit den Ansprüchen aus dem Straßenumfeld verträglich. Bei den anderen Ortsdurchfahrten (Mödling und Leobersdorf) zeigt sich hingegen, dass die tatsächliche Kfz-Verkehrsbelastung mit den Ansprüchen aus dem StraBenumfeld unverträglich ist. Für den Straßenabschnitt im Industriegebiet in Bad Vöslau ist die tatsächliche Kfz-Verkehrsbelastung der Straße mit den Ansprüchen aus dem Straßenumfeld gerade noch verträglich. 
In der Zusammenschau beider Bewertungen zeigt sich die Tendenz, dass bei den Straßenabschnitten, die eine eher geringe Einstufung der Automated Drivability, d. h. eine eher geringe Eignung für automatisierte Fahrzeuge aufweisen (Mödling und Leobersdorf), die tatsächliche Kfz-Verkehrsbelastung mit den Ansprüchen des Straßenumfelds unverträglich ist. Beim Straßenabschnitt in Gumpoldskirchen, der eine geringe bis mittlere Eignung für automatisierte Fahrzeuge aufweist, ist dies jedoch nicht in dem Ausmaß der Fall und die tatsächliche Kfz-Verkehrsbelastung ist mit den Ansprüchen des Straßenumfelds verträglich. Beim Straßenabschnitt in Bad Vöslau, der eine eher hohe Eignung für automatisierte Fahrzeuge aufweist, ist die tatsächliche Kfz-Verkehrsbelastung mit den Ansprüchen des Straßenumfelds gerade noch verträglich.

Für die Planung zeigt dies, dass die Bewertung der Eignung für automatisierte Fahrzeuge einen Beitrag leisten kann, um zu identifizieren, in welchen Gebieten automatisierte Fahrzeuge aus technologisch-infrastruktureller Sicht eher bzw. mit weniger Anpassungen im Straßenraum denkbar sind. Es sollte jedoch für mögliche Einsatzgebiete automatisierter Fahrzeuge überdies genau untersucht werden, ob die derzeit vorhandene Kfz-Verkehrsbelastung mit den Ansprüchen aus dem Straßenumfeld verträglich ist - hier zeigt sich in der Untersuchung der Straßenabschnitte, dass bereits die derzeitige Situation zum Teil problematisch ist. Ist sie mit den Ansprüchen aus dem Straßenumfeld tatsächlich nicht verträglich, sollte in diesen Bereichen von einem Einsatz automatisierter Fahrzeuge, der mit einer erhöhten bzw. zusätzlichen Verkehrsstärke und einer dichteren Fahrzeugfolge verbunden ist und die Durchlässigkeit des Straßenraums für andere Verkehrsteilnehmerlnnen noch weiter verringert, möglicherweise abgesehen werden.

Tabelle 18 (siehe folgende Seite) gibt hierzu nochmals einen Überblick zur Bewertung der straßenräumlichen Verträglichkeit der unterschiedlichen Straßenabschnitte unter Berücksichtigung einer Zunahme der Verkehrsleistung im Zuge von automatisierten Fahrzeugen. Die Metastudie von Soteropoulos et al. (2019b) beschreibt im Hinblick auf die Wirkungen bei der Verkehrsleistung - aufgrund von Verlagerungen von anderen Verkehrsmodi und Leerfahrten - bei privaten automatisierten Fahrzeugen Spannen zwischen +1 und +59 Prozent und bei automatisiertem Car-Sharing zwischen +8 und +80 Prozent. Eine Reduktion des Verkehrsaufwands in der Spanne von -25 bis -10 Prozent zeigt sich allein bei der Annahme eines sehr hohen Anteils von RideSharing und damit eines hohen Besetzungsgrades (vgl. Soteropoulos et al. 2019b: 40). Für eine genaue Abbildung der Zunahme in der Verkehrsleistung durch automatisierte Fahrzeuge in den jeweiligen Straßenabschnitten wäre ein Verkehrsnachfragemodell notwendig, um die jeweiligen Anwendungsfälle automatisierter Fahrzeuge wie private automatisierte Fahrzeuge oder automatisierte Fahrzeuge des Car- oder Ride-Sharing (und die damit verbundenen Annahmen) für die Straßenabschnitte in den Beispielgemeinden spezifisch zu modellieren. Da eine solche Modellierung im Rahmen dieser Untersuchung jedoch nicht möglich ist, werden für die Betrachtung in der Tabelle daher exemplarisch Zunahmen in der Verkehrsleistung von +5 bis +30 Prozent herangezogen und jeweils die Einstufung der Verträglichkeit betrachtet.

Es zeigt sich, dass bereits eine geringe Zunahme der Verkehrsbelastung (+5 Prozent) durch automatisierte Fahrzeuge beim Straßenabschnitt in Mödling mit den Ansprüchen aus dem StraBenumfeld völlig unverträglich ist. Dies ist unter Berücksichtigung einer höheren Zunahme der Verkehrsbelastung (+30 Prozent) ebenso beim Straßenabschnitt in Leobersdorf der Fall. Auch beim Straßenabschnitt in Bad Vöslau führt bereits eine Zunahme der Verkehrsbelastung durch automatisierte Fahrzeuge von 20 Prozent dazu, dass diese mit den Ansprüchen aus dem Straßenumfeld unverträglich ist. Allein beim Straßenabschnitt in Gumpoldskirchen zeigt sich, - aufgrund des geringen Ausgangswertes in der Verkehrsbelastung -, dass auch eine Zunahme der Verkehrsbelastung durch automatisierte Fahrzeuge um bis zu 30 Prozent mit den Ansprüchen aus dem Straßenumfeld verträglich ist. 
Tabelle 18: Bewertung der straßenräumlichen Verträglichkeit unter Berücksichtigung einer Zunahme der Verkehrsbelastung durch automatisierte Fahrzeuge

\begin{tabular}{|c|c|c|c|c|}
\hline $\begin{array}{c}\text { Straßenabschnitt, } \\
\text { Gemeinde }\end{array}$ & $\begin{array}{l}\text { Adaptierte } \\
\text { Gesamtwerte } \\
\text { (Fz/Sp-h) }\end{array}$ & $\begin{array}{c}\text { Zunahme der } \\
\text { Verkehrsbelastung } \\
\text { durch automatisierte } \\
\text { Fahrzeuge }\end{array}$ & $\begin{array}{l}\text { Verkehrs- } \\
\text { belastung } \\
\text { (Fz/Sp-h) }\end{array}$ & $\begin{array}{c}\text { Einstufung } \\
\text { Verträglichkeit }\end{array}$ \\
\hline \multicolumn{5}{|l|}{ Hauptstraße, Mödling } \\
\hline & $\begin{aligned} &<150(++) \\
& 150 \text { bis } 225(+) \\
&>225 \text { bis } 325(0) \\
&>325 \text { bis } 475(-) \\
&> 475(--)\end{aligned}$ & $\begin{array}{l} \pm 0 \% \\
+5 \% \\
+10 \% \\
+20 \% \\
+30 \%\end{array}$ & $\begin{array}{l}454 \\
477 \\
499 \\
545 \\
590\end{array}$ & $\begin{array}{c}\text { - unverträglich } \\
\text { - - völlig unverträglich } \\
\text { - - völlig unverträglich } \\
\text { - - völlig unverträglich } \\
\text { - - völlig unverträglich }\end{array}$ \\
\hline
\end{tabular}

Wiener Straße, Gumpoldskirchen
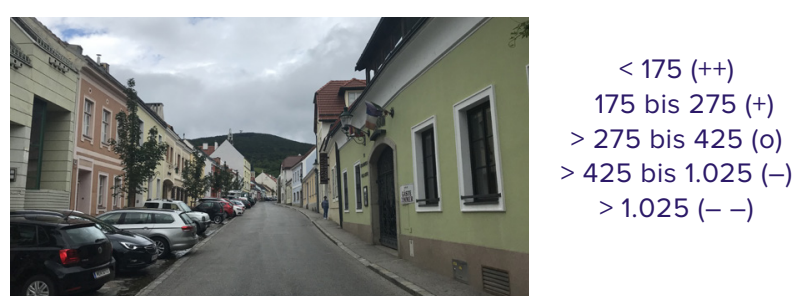

$\pm 0 \%$

$+5 \%$

$+10 \%$

$+20 \%$

$+30 \%$

$\begin{array}{lc}158 & ++ \text { gut verträglich } \\ 166 & ++ \text { gut verträglich } \\ 174 & ++ \text { gut verträglich } \\ 190 & + \text { verträglich } \\ 205 & + \text { verträglich }\end{array}$

Industriestraße, Bad Vöslau

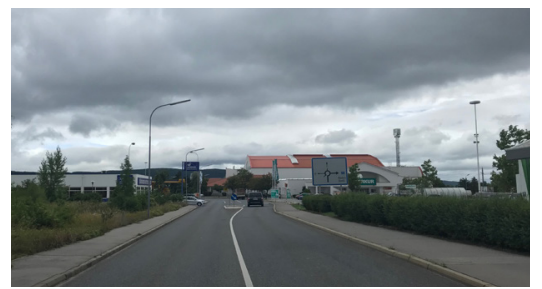

$$
<400(++)
$$

400 bis $1.000(+)$

$>1.000$ bis 1.200 (o)

$>1.200$ bis $1.500(-)$

$$
>1.500(--)
$$

o gerade noch verträglich

o gerade noch verträglich

o gerade noch verträglich

- unverträglich

- unverträglich

Hauptstraße, Leobersdorf

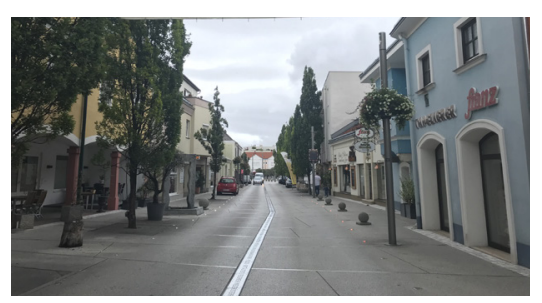

$$
\begin{gathered}
<325(++) \\
325 \text { bis } 400(+) \\
>400 \text { bis } 500(0) \\
>500 \text { bis } 650(-) \\
>650(--)
\end{gathered}
$$

$\pm 0 \%$

$+5 \%$

$+10 \%$

$+20 \%$

$+30 \%$

$$
\begin{aligned}
& \pm 0 \% \\
& +5 \% \\
& +10 \% \\
& +20 \% \\
& +30 \%
\end{aligned}
$$

512

538

563

614

666
- unverträglich

- unverträglich

- unverträglich

- unverträglich

- - völlig unverträglich

Ist in Straßenräumen die derzeit vorhandene Kfz-Verkehrsbelastung mit den Ansprüchen aus dem Straßenumfeld nicht verträglich, kann somit davon ausgegangen werden, dass - abhängig von der jeweiligen Zunahme der Verkehrsbelastung durch automatisierte Fahrzeuge - der Einsatz automatisierter Fahrzeuge dazu führt, dass die Straßenräume noch unverträglicher mit den Ansprüchen aus dem Straßenumfeld werden. Hier sollte der Straßenraum stattdessen verträglicher für die Ansprüche aus dem Straßenumfeld gestaltet werden (z. B. Verringerung 
der Geschwindigkeit, weitere Übergangshilfen zur direkten Querung, Umnutzung von Parkplatzflächen) bzw. der Einsatz automatisierter Fahrzeuge an diese Maßnahmen gekoppelt sein (vgl. Anciaes/Jones 2016: 4). Um darüber hinaus auch die Zunahme bei der Verkehrsleistung durch automatisierte Fahrzeuge zu verringern, sind ebenso Maßnahmen wie ein dynamisches „Road Pricing“ bzw. „Mobility Pricing“, d. h. eine Kontingentierung von Fahrzeugen bzw. gefahrenen Kilometern in bestimmten Abschnitten, oder Maßnahmen zur Erhöhung des Besetzungsgrades (z. B. Verbote oder Abgabe für Leerfahrten) sinnvoll (vgl. Soteropoulos et al. 2019a: 133). Auch der Einsatz automatisierter Fahrzeuge im öffentlichen Verkehr kann dienlich sein, es sollte jedoch genau abgewogen werden, ob dies - insbesondere auch wenn baulich-infrastrukturelle Anpassungen nötig sind - mit den Ansprüchen aus dem Straßenumfeld verträglich ist. Zum Zusammenhang von der Eignung von Straßenräumen für den Einsatz automatisierter Fahrzeuge aus technologisch-infrastruktureller Sicht und der Verträglichkeit von Straßenräumen wären hierbei weitere umfassende Untersuchungen von Bedeutung.

Zusätzlicher Forschungsbedarf besteht auch im Hinblick darauf, inwieweit Adaptierungen zur Verbesserung der straßenräumlichen Verträglichkeit oder Maßnahmen zur Ermöglichung bestimmter Anwendungsfälle automatisierten Fahrens (wie etwa Hop-On-Hop-Off-Bereiche für das Ein- und Aussteigen in automatisierte Fahrzeugflotten; vgl. Beitrag 8 von Bruck et al. in diesem Band) auf die Eignung dieser Straßenräume für automatisierte Fahrsysteme und umgekehrt wirken. Bei den Ergebnissen des durchgeführten Verfahrens zur Bewertung der straßenräumlichen Verträglichkeit sollten jedoch mehrere Aspekte berücksichtigt werden bzw. besteht weiterer Forschungsbedarf:

a. Die Zählungen des Kfz-Verkehrs sowie von Fußgängerlnnen und RadfahrerInnen an den Straßenabschnitten müssten in umfassenderer Weise durchgeführt werden, um die Erkenntnisse weiter zu schärfen ${ }^{3}$. Einerseits wurden die Verkehrszählungen im November durchgeführt, einem Monat, in welchem aus saisonalen Gründen deutlich weniger RadfahrerInnen und FußgängerInnen in den jeweiligen Straßenabschnitten unterwegs sind als zu anderen Jahreszeiten. Andererseits fand die Verkehrszählung nicht für alle VerkehrsteilnehmerInnen zur jeweiligen Spitzenstunde statt (z. B. FußgängerInnen 12 bis $14 \mathrm{Uhr}$ und 16 bis $18 \mathrm{Uhr}$, Radfahrerlnnen 12 bis $14 \mathrm{Uhr}$, Kfz-Verkehr 7 bis $11 \mathrm{Uhr}$ und 15 bis 19 Uhr; vgl. FGSV 2012: 28), sondern wurde jeweils im Zeitraum von 15 bis 16 Uhr durchgeführt, sodass die Ergebnisse der Verkehrszählung vom tatsächlichen Aufkommen der VerkehrsteilnehmerInnen in der jeweiligen Spitzenstunde abweichen können. Es ist deshalb davon auszugehen, dass sich die Bewertung der straßenräumlichen Verträglichkeit bei einigen der untersuchten Straßenabschnitte tendenziell etwas schlechter darstellt, da vermutlich jeweils mehr VerkehrsteilnehmerInnen in den jeweiligen Spitzenstunden unterwegs sind, als im Rahmen der derzeitigen Bewertung berücksichtigt wurde. Neben umfangreicheren manuellen Verkehrszählungen könnten hierbei auch automatische Verkehrszählungen, beispielsweise durch Seitenradargeräte, zum Einsatz kommen und das tatsächliche Verkehrsaufkommen an den Straßenquerschnitten deutlich genauer abbilden (vgl. FGSV 2012: 35). Zudem müssten auch die aus der GIP abgeleiteten Werte für die v85-Geschwindigkeit der Fahrzeuge bei den Straßenabschnitten im Hinblick auf ihre Validität überprüft werden. Hier geht es mittels umfangreicher Geschwindigkeitsmessungen auch darum, gewisse Varianzen in den gefahrenen Geschwindigkeiten miteinzuschließen (z. B. unterschiedliche Tageszeit: höhere Fahrzeugdichte und geringere Geschwindigkeiten zu Stoßzeiten, höhere Geschwindigkeiten in der Nacht), um so die v85-Geschwindigkeit der Fahrzeuge detaillierter abzubilden.

3 Die Verkehrszählungen zur Ermittlung der Anzahl der Fahrzeuge in der Spitzenstunde, der Anzahl von Fußgängerlnnen und Radfahrerlnnen sowie des Lkw-Anteils wurden von Michael Haudum im Rahmen seiner Diplomarbeit durchgeführt. 
b. Der Festlegung des Grundwerts der verträglichen Verkehrsbelastung kommt im Rahmen des Verfahrens zur Bewertung der straßenräumlichen Verträglichkeit eine besonders bedeutende Rolle zu. Zwar wurde bei der Festsetzung auf die in der Studie von Mörner et al. (1984) sowie Bühlmann und Laube (2013) verwendeten Grundwerte zurückgegriffen und diese geringfügig adaptiert, sinnvoll ist es jedoch, mit Hilfe von weiteren exemplarischen Straßenabschnitten eine Sensitivitätsanalyse hinsichtlich der Grundwerte durchzuführen.

c. Der zur Bewertung der straßenräumlichen Verträglichkeit verwendete Kompensatorische Ansatz berücksichtigt zahlreiche Aspekte nur unzureichend, die jedoch im Hinblick auf eine Bewertung der straßenräumlichen Verträglichkeit von Relevanz wären. Einerseits fehlen relevante Faktoren wie beispielsweise die Barrierefreiheit für Fußgängerlnnen gänzlich. Wenngleich sich hinsichtlich der Berücksichtigung zusätzlicher Aspekte immer ein Spannungsfeld zwischen der Anwendbarkeit des Ansatzes aufgrund verfügbarer Daten und der Detaillierungstiefe des Ansatzes bzw. der Anzahl der berücksichtigten Aspekte bezüglich der straßenräumlichen Verträglichkeit ergeben, scheint die Berücksichtigung von einigen weiteren Faktoren durchaus von Relevanz, um die straBenräumliche Verträglichkeit umfassender abzubilden. Zur Berücksichtigung weiterer Gesichtspunkte können hierbei auf die von Gehl Architects (2009) beschriebenen Qualitätskriterien für den öffentlichen Raum zurückgegriffen werden bzw. können im Nachgang zur Bewertung der straßenräumlichen Verträglichkeit im Sinne eines zweistufigen Verfahrens zudem die von Gehl Architects beschriebenen Kriterien bewertet werden (vgl. Gehl Architects 2009: 43).

d. Andererseits werden Aspekte wie beispielsweise (1) die Flächenaufteilung zwischen Kfz und Fußgängerlnnen bzw. Radfahrerlnnen oder (2) Grünflächen und Gestaltungselemente im Straßenraum aufgrund der Logik des Kompensatorischen Ansatzes jeweils nur als ein Kriterium von mehreren berücksichtigt, anhand derer auch nur eine Kompensation der verträglichen Verkehrsbelastung vorgenommen wird. Die Ergebnisse für den Straßenabschnitt in Gumpoldskirchen - hier finden sich Gehsteigbreiten, die sich an der Grenze der Mindeststandards bewegen (vgl. FSV 2015), im Ergebnis ist jedoch eine gute Verträglichkeit ersichtlich - zeigen jedoch, dass Themen wie eine ausreichende Gehsteigbreite für Fußgängerlnnen im Rahmen des Ansatzes nur unzureichend berücksichtigt werden. Eine Adaptierung des Kompensatorischen Ansatzes mit einer höheren Gewichtung (höhere Kompensationswerte) erscheint bezüglich der Flächenaufteilung zwischen Kfz und Fußgängerlnnen bzw. Radfahrerlnnen von Wichtigkeit. Dies gilt auch in Bezug auf Grünflächen, denen vor dem Hintergrund des Klimawandels und ihrer Möglichkeiten, Hitzeinselbildungen entgegenzuwirken (vgl. Sandholz/Sett 2019: 11), eine größere Bedeutung bei der Bewertung der straßenräumlichen Verträglichkeit zukommen sollte.

e. Vor dem Hintergrund der Bewertung der straßenräumlichen Verträglichkeit im Zusammenhang mit dem automatisierten Fahren sollte das Verfahren stärker mit den Wirkungen automatisierter Fahrzeuge verknüpft werden bzw. umfassender um diesbezügliche Aspekte erweitert werden. Ein Beispiel hierfür ist die mit der dichteren Fahrfolge von automatisierten Fahrzeugen verbundene Trennwirkung für den Straßenraum. Im Rahmen dieses Artikels wurde das Verfahren bereits um die Berücksichtigung von Querungshilfen erweitert. Weitere Berücksichtigung sollte jedoch beispielsweise auch die Bewertung des Querungsbedürfnisses finden. Hier geht es durch eine detaillierte Untersuchung des Querungsverhaltens von FußgängerInnen und Radfahrerlnnen darum, abzuschätzen, ob es aus Sicht der Fußgängerlnnen und Radfahrerlnnen ein punktuelles Querungsbedürfnis gibt, für das punktuelle Querungsstellen ausreichen oder ein lineares Querungsbedürfnis (z. B. in Geschäftsstraßen) oder ein flächiges (z. B. an Bahnhofsvorplätzen) vorliegt (vgl. Häfliger et al. 2015: 80). Hier gilt auch zu berücksichtigen, dass gemäß FGSV (2002) bei Straßen mit zwei Fahrstreifen bis 8,50 Meter Fahrbahnbrei- 
te eine Querung durch FußgängerInnen kaum möglich ist, wenn (1) die Verkehrsstärke mehr als $1.000 \mathrm{Kfz} / \mathrm{Sp}$-h und die Geschwindigkeit 50 km/h beträgt oder wenn (2) die Verkehrsstärke mehr als $500 \mathrm{Kfz} / \mathrm{Sp}$-h und die Geschwindigkeit mehr als 50 km/h beträgt. In diesen beiden Fällen werden in jedem Fall Querungsanlagen benötigt (vgl. FGSV 2002). Gleichfalls wäre in diesem Zusammenhang auch eine Bewertung der Wartezeit bei Querungshilfen (wie Verkehrslichtsignalanlagen und Schutzwegen) von Relevanz.

f. Eine umfassendere Berücksichtigung dieser Kriterien ist von besonderer Bedeutung, nicht nur um abzuschätzen, wie viel Mehrverkehr durch automatisierte Fahrzeuge für den Straßenraum verträglich ist, sondern inwiefern auch die die Kapazitätssteigerung ermöglichende dichtere Fahrzeugfolge von automatisierten Fahrzeugen mit dem StraBenumfeld verträglich ist und welche Maßnahmen und Adaptierungen nötig sind ( $z$. B. eine etwaige Kanalisierung von FußgängerInnen, die die Fahrbahn queren), um die Wirkungen der Kapazitätssteigerung und der Trennwirkung bestmöglich auszutarieren.

g. Letztlich sollte noch umfassender untersucht werden, welche nicht per se verkehrlich intendierten Wirkungen durch automatisierte Fahrzeuge darüber hinaus deren Verträglichkeit mit den Umfeldnutzungen beeinflussen könnten; der Ansatz der straßenräumlichen Verträglichkeit sollte dahingehend erweitert werden. Beispielsweise erfassen schon heute jene allein mit Technik zum automatisierten Fahren ausgestatteten Fahrzeuge (z. B. Tesla Autopilot) durch ihre Sensoren die Umgebung und damit auch unweigerlich entsprechende Nutzungen sowie Personen und deren Aktivitäten im Straßenraum. Eine solche „Iaufende“ Erfassung von Daten und Personen bzw. Überwachung durch automatisierte Fahrzeuge scheint für manche Nutzungen und Aktivitäten möglicherweise unproblematisch, für andere sensible Anwendungen, wie beispielsweise vor dem Hintergrund politischer Kundgebungen o. Ä. (vgl. Heger 2008: 93), ist jedoch zu klären, ob und in welchem Ausmaß diese Datenerfassung stattfinden kann (Stichwort Privatheit). Es geht also um die Frage, inwieweit automatisierte Fahrzeuge mit solchen Nutzungen „verträglich“ sind (vgl. Beitrag 10 von Mitteregger in diesem Band). Eine Erweiterung der straßenräumlichen Verträglichkeit durch zusätzliche Aspekte scheint somit im Zusammenhang mit automatisierten Fahrzeugen von Relevanz und sollte näher untersucht werden.

\section{LITERATUR}

Abegg, C., C. Girod, K. Fischer, N. Pahud, L. Raymann und F. Perret 2018. „Einsatz automatisierter Fahrzeuge im Alltag - Denkbare Anwendungen und Effekte in der Schweiz. Schlussbericht Modul 3d ,Städte und Agglomerationen“", Fassung vom 30.8.2018. www.ebp.ch/sites/default/files/project/uploads/2018-08-30\%20aFn_3d\%20St\%C3\%A4dte-Agglomerationen\%20Schlussbericht_1. pdf (4.5.2020).

Anciaes, P., und P. Jones 2016. „How do pedestrians balance safety, walking time, and the utility of crossing the road? A stated preference study", Street Mobility and Network Accessibility Series, Working Paper 8. London: UCL. https://pdfs.semanticscholar.org/bac7/2aa3228d282fe2cdf260ac4388be21b23dd5.pdf (6.5.2020).

Baier, R. 1992. „Verträglichkeit des Kraftfahrzeugverkehrs in Straßenräumen und Straßennetzen - Praxisorientiertes Verfahren in der Verkehrsentwicklungsplanung“, in Internationales Verkehrswesen 10, 395-399.

Baier, R., und FGSV (Forschungsgesellschaft für Straßen- und Verkehrswesen) 2007. Richtlinien für die Anlage von Stadtstraßen - RASt 06, hg. v. FGSV - Forschungsgesellschaft für Straßen- und Verkehrswesen. Köln: FGSV Verlag. 
Baier, R., C. Hebel, Y. Jachtmann, A. Reinartz, K.-H. Schäfer und A. Warnecke 2011. „Stadt Mönchengladbach. Untersuchungen zur Verkehrsentwicklungsplan“, hg. v. BSV - Büro für Stadt- und Verkehrsplanung Reinhold Baier, Aachen. https://tinyurl.com/yaqvgpwk (4.5.2020).

BMVIT (Ministerium für Verkehr, Innovation und Technologie) 2018. „Testberichte. Tests auf Straßen mit öffentlichem Verkehr in Österreich gemäß der Automatisiertes Fahren Verordnung. Zeitraum: 20162018“, Wien. https://bit.ly/3ereyGW (4.5.2020).

Brummelen, J. van, M. O’Brien, D. Gruyer und H. Najjaran 2018. „Autonomous vehicle perception: The technology of today and tomorrow“, in Transportation Research Part C: Emerging Technologies 89, 384-406.

Bühlmann, F., und M. Laube 2013. „Verträglichkeit Strassenraum. Methodik und Ergebnisse“, hg. v. Kanton Zürich. Zürich: Amt für Verkehr. https://tinyurl.com/y7nou6qc (4.5.2020).

Czarnecki, K. 2018. „Operational Design Domain for Automated Driving Systems. Taxonomy of Basic Terms“, Waterloo Intelligent Systems Engineering (WISE) Lab, University of Waterloo, Canada.

Favaro’, F. M., S. Eurich und N. Nader 2018. „Autonomous vehicles' disengagements: Trends, triggers, and regulatory limitations“, in Accident Analysis and Prevention 110, 136-148.

FGSV (Forschungsgesellschaft für Straßen- und Verkehrswesen) 2002. Empfehlungen für Fußgängerverkehrsanlagen (EFA). Köln: FGSV Verlag.

FGSV (Forschungsgesellschaft für Straßen- und Verkehrswesen) 2012. EVE - Empfehlungen für Verkehrserhebungen. Köln: FGSV Verlag.

Frehn, M., G. Steinberg und S. Schröder 2013. „Methodik und Ergebnisse der Straßenraumverträglichkeit. Verkehrsentwicklungsplan Bremen 2025“. www.bau.bremen.de/sixcms/media.php/13/130228_ E03_Strassenraumvertraeglichkeit_Methodik_Ergebnisse.pdf (5.5.2020).

FSV (Forschungsgesellschaft Straße - Schiene - Verkehr) 2015. „RVS 03.02.12 Fußgängerverkehr“. Wien.

Gehl Architects 2009. „Downtown Seattle: Public Spaces \& Public Life“, hg. v. City of Seattle. www.seattle.gov/Documents/Departments/SDCI/Codes/PublicSpacesLifelntro.pdf (7.5.2020).

GIP.at 2019. „Intermodaler Verkehrsgraph Österreich. Standardbeschreibung der Graphenintegrationsplattform (GIP), Version 2.3“. www.gip.gv.at/assets/downloads/GIP_Standardbeschreibung_2.3.pdf (5.5.2020).

Häfliger, R., J. Bubenhofer, C. Hagedorn, K. Zweibrücken, S. Condrau und R. Baier 2015. „Verträglichkeitskriterien für den Strassenraum innerorts. Forschungsprojekt SVI 2004/058 auf Antrag der Schweizerischen Vereinigung der Verkehrsingenieure und Verkehrsexperten (SVI)“. Zürich: Schweizerischer Verband der Strassen- und Verkehrsfachleute (VSS).

Heger, Nora 2008. „Entgrenzte Räume. Kontrolle des öffentlichen Raums am Beispiel der Videoüberwachung am Wiener Schwedenplatz“, Diplomarbeit, Universität Wien. http://othes.univie. ac.at/2690/1/2008-10-21_0002326.pdf (7.5.2020).

Heinrichs, D. 2015. „Autonomes Fahren und Stadtstruktur“, in Autonomes Fahren. Technische, rechtliche und gesellschaftliche Aspekte, hg. v. M. Maurer, J. C. Gerdes, B. Lenz und H. Winner. Berlin/Heidelberg: Springer Vieweg, 219-239.

Hörl, S., F. Becker, T. Dubernet und K. W. Axhausen 2019. „Induzierter Verkehr durch autonome Fahrzeuge: Eine Abschätzung“. Bern: Bundesamt für Strassen. https://ethz.ch/content/dam/ethz/specialinterest/baug/ivt/ivt-dam/vpl/reports/1401-1500/ab1433.pdf (4.5.2020).

Litman, T. A. 2009. „Barrier Effect“, in Transportation Cost and Benefit Analysis. Techniques, Estimates and Implication, 2. Aufl., hg. v. T. A. Litman. Victoria: Victoria Transport Policy Institute, Kap. 5.13. www. vtpi.org/tca/tca0513.pdf (4.5.2020).

Marsden, G., J. Dales, P. Jones, E. Seagriff und N. Spurling 2018. „All change? The future of travel demand an the implications for policy and planning. The First Report of the Commission on Travel Demand“. www.demand.ac.uk/wp-content/uploads/2018/04/FutureTravel_report_final.pdf (4.5.2020).

Marshall, S. 2005. Street \& Patterns. London: Spon Press.

Milakis, D., B. van Arem und B. van Wee 2017. „Policy and society related implications of automated driving: A review of literature and directions for future research“, in Journal of Intelligent Transportation Systems. Technology. Planning and Operations (21) 4, 324-348.

Mörner, J. von, P. Müller und H. Topp 1984. „Forschung Strassenbau und Strassenverkehrstechnik, Entwurf und Gestaltung innerörtlicher Strassen“, Bericht 425 der Schriftenreihe Forschung Straßenbau und Straßenverkehrstechnik. Bonn: Bundesministerium für Verkehr. 
Müller, P., H.-J. Collin, A. Ratschow und W. Rüthrich 1994. „Das LADIR-Verfahren zur Bestimmung stadtverträglicher Belastungen durch Autoverkehr“, Schlussbericht zum Forschungsprojekt des Forschungsfeldes „Städtebau und Verkehr“ im Bundesministerium für Raumordnung, Bauwesen und Städtebau, Darmstadt/Braunschweig.

Nørby, L. E., und K. R. Meltofte 2012. „Over Vejen. Vejen som trafikal barriere for fodgængere“. Aalborg: Aalborg Universitet. https://projekter.aau.dk/projekter/files/63452678/Over_vejen_hovedrapport_. pdf (4.5.2020).

OECD 2020. „Functional urban areas by country“. www.oecd.org/cfe/regional-policy/functionalurbanareasbycountry.htm (5.5.2020).

Pendleton, S. D., H. Andersen, X. Du, X. Shen, M. Meghjani, Y. H. Eng, D. Rus und M. H. Ang Jr. 2017. „Perception, Planning, Control, and Coordination for Autonomous Vehicles“, in Machines (5) 6, 1-54.

SAE International 2018. „Taxonomy and Definitions for Terms Related to Driving Automation Systems for On-Road Motor Vehicles - J3016“, Juni 2018. www.sae.org/standards/content/j3016_201806/ (4.5.2020).

Sandholz, S., und D. Sett 2019. „Erfahrungen und Bedarfe von Akteuren der Stadtplanung im Hinblick auf Vulnerabilität gegenüber Hitzestress. Ergebnisse einer Haushalts-Umfrage zum Hitzeempfinden in Bonn“, ZURES Working Paper 2, 08/2019. https://collections.unu.edu/eserv/UNU:7510/ZURES_workingpaper2_ErgebnisseHHUmfrage_UNU-EHS-1.pdf (7.5.2020).

Shladover, S. E. 2018a. „Connected and automated vehicle systems: Introduction and overview“, in Journal of Intelligent Transportation Systems (22) 3, 190-200.

Shladover, S. E. 2018b. „Practical Challenges to Deploying Highly Automated Vehicles“, Präsentation bei Drive Sweden, Göteborg.

Soteropoulos, A., A. Stickler, V. Sodl, M. Berger, J. Dangschat, P. Pfaffenbichler, G. Emberger, E. Frankus, R. Braun, F. Schneider, S. Kaiser, H. Walkobinger und A. Mayerthaler 2019a. „SAFiP - Systemszenarien Automatisiertes Fahren in der Personenmobilität. Endbericht“. Wien: Bundesministerium für Verkehr, Innovation und Technologie. https://projekte.ffg.at/anhang/5cee1b11a1eb7_SAFiP_Ergebnisbericht.pdf (4.5.2020).

Soteropoulos, A., M. Berger und F. Ciari 2019b. „Impacts of automated vehicles in travel behaviour and land use: An international review of modelling studies“, in Transport Reviews (39) 1, 29-49.

Soteropoulos, A., M. Mitteregger, M. Berger und J. Zwirchmayr 2020. „Automated drivability: Toward an assessment of the spatial deployment of level 4 automated vehicles", in Transportation Research Part A: Policy and Practice 136, 64-84.

Stadt Wien 2018. „STEP 2025. Fachkonzept Öffentlicher Raum“, hg. v. Stadtentwicklung Wien, Magistratsabteilung 18 - Stadtentwicklung und Stadtplanung. www.wien.gv.at/stadtentwicklung/studien/pdf/ b008522.pdf (4.5.2020).

Su, S., H. Zhou, M. Xu, H. Ru, W. Wang und M. Wenig 2019. „Auditing street walkability and associated social inequalities for planning implications“, in Journal of Transport Geography 74, 62-76.

Wissenschaftlicher Beirat beim Bundesminister für Verkehr und digitale Infrastruktur 2017. „Automatisiertes Fahren im Straßenverkehr. Herausforderungen für die zukünftige Verkehrspolitik“. Berlin: Bundesministerium für Verkehr und digitale Infrastruktur. www.bmvi.de/SharedDocs/DE/Anlage/G/ wissenschaftlicher-beirat-gutachten-2017-1.pdf?__blob=publicationFile (4.5.2020).

Open Access Dieser Beitrag wird unter der Creative Commons Namensnennung 4.0 International Lizenz (http://creativecommons.org/licenses/by/4.0/deed.de) veröffentlicht, welche die Nutzung, Vervielfältigung, Bearbeitung, Verbreitung und Wiedergabe in jeglichem Medium und Format erlaubt, sofern Sie den/die ursprünglichen Autor(en) und die Quelle ordnungsgemäß nennen, einen Link zur Creative Commons Lizenz beifügen und angeben, ob Änderungen vorgenommen wurden.

Die in diesem Beitrag enthaltenen Bilder und sonstiges Drittmaterial unterliegen ebenfalls der genannten Creative Commons Lizenz, sofern sich aus der Abbildungslegende nichts anderes ergibt. Sofern das betreffende Material nicht unter der genannten Creative Commons Lizenz steht und die betreffende Handlung nicht nach gesetzlichen Vorschriften erlaubt ist, ist für die oben aufgeführten Weiterverwendungen des Materials die Einwilligung des jeweiligen Rechteinhabers einzuholen.

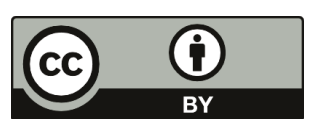

